\title{
Applying kriging proxies for Markov chain Monte Carlo in reservoir simulation
}

\author{
Ilya Fursov ${ }^{1} \cdot$ Mike Christie $^{1} \cdot$ Gabriel Lord ${ }^{1}$
}

Published online: 13 June 2020

(C) The Author(s) 2020

\begin{abstract}
One way to quantify the uncertainty in Bayesian inverse problems arising in the engineering domain is to generate samples from the posterior distribution using Markov chain Monte Carlo (MCMC) algorithms. The basic MCMC methods tend to explore the parameter space slowly, which makes them inefficient for practical problems. On the other hand, enhanced MCMC approaches, like Hamiltonian Monte Carlo (HMC), require the gradients from the physical problem simulator, which are often not available. In this case, a feasible option is to use the gradient approximations provided by the surrogate (proxy) models built on the simulator output. In this paper, we consider proxy-aided HMC employing the Gaussian process (kriging) emulator. We overview in detail the different aspects of kriging proxies, the underlying principles of the HMC sampler and its interaction with the proxy model. The proxy-aided HMC algorithm is thoroughly tested in different settings, and applied to three case studies — one toy problem, and two synthetic reservoir simulation models. We address the question of how the sampler performance is affected by the increase of the problem dimension, the use of the gradients in proxy training, the use of proxy-for-the-data and the different approaches to the design points selection. It turns out that applying the proxy model with HMC sampler may be beneficial for relatively small physical models, with around 20 unknown parameters. Such a sampler is shown to outperform both the basic Random Walk Metropolis algorithm, and the HMC algorithm fed by the exact simulator gradients.
\end{abstract}

Keywords Markov chain Monte Carlo · Uncertainty quantification · Gaussian process · Proxy model $\cdot$ Reservoir simulation

Mathematics subject classification (2010) 65C40, 60G15, 68U20

\section{Introduction: HMC samplers and proxy models}

One of the common ways to tackle an inverse problem is finding a single model which is calibrated (history matched) to reproduce the observation data. A more advanced approach involves quantification of uncertainties (UQ), e.g. by generating a range of models consistent both with the prior information, and with the observed data, or, strictly speaking, sampling from the target posterior distribution. This is usually done by employing a Markov chain Monte Carlo (MCMC) sampler. Sampling from the posterior target and UQ is more challenging and computationally demanding than the mere

Ilya Fursov

ilya.foursov@mail.ru

1 Heriot-Watt University, Edinburgh, UK model calibration, but may be more appropriate, since having the information on the uncertainties of the simulation model allows a higher quality decision making.

An MCMC sampler generates a Markov chain-i.e. a sequence of points/models $\left\{x_{i}\right\}$ where a new model $x_{i+1}$ is found based only on the current model $x_{i}$, and does not depend on the preceding models $x_{i-1}, x_{i-2}, \ldots$ given $x_{i}$. A properly designed MCMC sampler, after some initial iterations (called the burn-in stage), reaches the stationarity stage, where the generated points $\left\{x_{i}\right\}$ are distributed according to the desired target distribution (or at least a distribution asymptotically approaching the target distribution). At the same time, these points $\left\{x_{i}\right\}$ will usually be mutually correlated, which is a drawback of the MCMC algorithms. Producing a Markov chain with high correlations (also referred to as slow chain mixing) leads to inefficient sampling, since a much larger chain length will be required to get any useful information from it.

A typical (Metropolis-Hastings) MCMC algorithm involves two key steps in each iteration: (1) making a proposal 
move, and (2) accepting or rejecting the proposal with certain probability. For a high performance MCMC sampler, one would require both the proposals that explore more fully the parameter space, and a high probability of accepting these proposals.

With an increase in the dimension of the inverse problem, simple MCMC samplers like the basic Random Walk Metropolis (RWM) algorithm [34] suffer from slow chain mixing. As a consequence, their efficiency drops, and a large number of the forward model runs may be required (from thousands to millions), making them impractical for the realistic problems. To tackle this, more advanced samplers can be used, like Hamiltonian Monte Carlo (HMC), which allows non-local proposals for the Markov chain, at the same time maintaining high acceptance rates.

However, in order for HMC to work, gradients of the log likelihood function are required. ${ }^{1}$ When the model in question is a multiphase flow in porous medium, the simulators may not provide these gradients. In this situation, application of the $\mathrm{HMC}$ is not possible, and this brings us to consider building a proxy model for the reservoir simulation model, from which approximate gradients can be found.

Proxy models (in this work, we use this shorter term as a synonym of the surrogate models or response surfaces or emulators) have been used as cheaper alternatives to the fullfledged simulation models in a variety of applications; we provide a short, inexhaustive review of them. Zubarev [40] investigated the performance of different proxy types (polynomial response surfaces, kriging, thin-plate splines, artificial neural networks) applied to history matching of the reservoir simulation problems. Lan, Bui-Thanh [24] employed the Gaussian process (kriging) emulator for approximating the higher order derivatives involved in the manifold MCMC sampling for Bayesian inverse problems. Jones [21] discussed different approaches for global optimisation aided by kriging response surfaces, using the statistical error estimates provided by the kriging machinery. Another approach for optimisation that relies on GP, and allows incorporation of the prior data, noise, and gradients for the objective function, is tackled by Osborne [32].

Christen and Sanso [9] applied the Gaussian process emulator while investigating the approaches for design of computer experiments for MIT2D climate model. Gramacy and Lee [17] investigated how nugget can enhance applicability of Gaussian process (GP) model and treed Gaussian process (TGP) model for emulating deterministic computer experiments. In particular, they considered application of GP and TGP to the Langley Glide-Back Booster CFD computer experiments. The treed Gaussian process [16] was designed to

\footnotetext{
${ }^{1}$ More sophisticated samplers, like the Manifold HMC [24], require higher order derivatives in addition to the gradients.
}

handle the non-stationary data, by partitioning these data, and fitting separate stationary GPs to them.

In the work presented here, we use proxies based on kriging, or Gaussian process emulators [35], and the reader is referred to Appendix A for the technical details and the relevant references from the literature. A kriging proxy essentially works as an interpolator ${ }^{2}$ for some real-valued function $g(x)$ defined on a $d$-dimensional parameter space $\mathbb{R}^{d}$. It is trained based on the responses of $g(x)$ at the design points $x_{1}, \ldots, x_{n}$, and makes use of the covariance function defined in $\mathbb{R}^{d}$. This covariance function, provided by the user, specifies the anticipated degree of correlation of the responses at different points $x_{i}, x_{j}$. In addition, kriging may use trend functions, e.g. constant, linear or quadratic trends, which may be beneficial if $g(x)$ can be reasonably approximated by them. In this case, the proxy would essentially work in two stages, first fitting the combination of the trend functions (trend surface) to the responses of $g(x)$ at the design points (although this fit depends on the covariances), and then performing the pure kriging interpolation for the residual.

For history-matching (model calibration) applications, one would need a proxy model to approximate the objective function (misfit), likewise for MCMC applications, a proxy for the log likelihood would be required. However, both misfit and log likelihood usually involve some combinations of the simulator responses at the observation points $i$, e.g. they can be defined as sum of squares involving the simulator responses $d(x)_{i}$ and the observed data $d_{o, i}$ :

$f(x)=\sum_{i}\left(\frac{d(x)_{i}-d_{o, i}}{\sigma_{i}}\right)^{2}$

It then makes sense not to build a proxy for the misfit $f(x)$, but instead to construct multiple proxies for the simulator responses $d(x)_{i}$ at all data points $i$, and then substitute them into the misfit formula (1). Taking this step results in a "greybox" rather than an entirely black-box approach, and the proxy model is thus freed from the need to handle the nonlinearities introduced by the quadratic misfit formula, potentially increasing the overall proxy accuracy. This idea is certainly not new, e.g. He, Xie [19] showed the accuracy benefits of proxy-for-data over the proxy-for-mismatch. The multi-output Gaussian process regression provides similar approaches to deal with the multidimensional simulator output, see e.g.

\footnotetext{
${ }^{2}$ Apart from the mean (the interpolator), GP machinery also provides the associated variance, i.e. it gives the estimate of the anticipated error of prediction. We note that this estimate may not be appropriate, for example, if the underlying function $g(x)$ does not have reasonable stationarity, or is otherwise not suitable to be approximated by a stationary Gaussian process. On the other hand, the interpolation property of the GP mean is achievable in most situations (except, e.g., numerically degenerate cases or the non-zero nugget cases), for a wide class of the underlying functions (here, we also note that interpolation may not mean good prediction). In this work, we do not employ the error estimates provided by the GP.
} 
Bilionis and Zabaras [5], and the references therein for the earlier work on this topic.

In our paper, the kriging proxy models built directly for the misfit $f(x)$ are called simple proxies, and the proxy models involving the kriging sub-proxies for the simulator output data $d(x)_{i}$ are called data proxies, see Appendix A, section data proxy for the formal definition. Our approach appears to be overall similar to that of Bilionis and Zabaras [5], i.e. using the same covariance matrix for all outputs (data points). For the case when the number of data points with available observations is high, construction and application of a data proxy may be computationally expensive. $\mathrm{He}$, Xie [19] handle this by using reduced order modelling. In our work, we instead use parallelisation via MPI, since running the subproxies for the numerous data points can be easily distributed across the different parallel processes, and the results are only collected together when calculating the final misfit or its derivatives.

Good design of the set of points used for the proxy model training is beneficial for accuracy. In this work, a two-stage design is employed: firstly, a uniform Sobol design is taken for the initial proxy training before the start of MCMC sampling. Then, during the burn-in stage of the MCMC, additional training points (those visited by the sampler) are periodically added to the proxy model. Only a fraction of the available training points is taken on each proxy update event, since kriging can hardly handle more than a few thousand training points in total. Yet, the training points are selected so as to provide "maximum information" on the underlying target function. Although we do not treat the "maximum information" goal in a formalised way, the main heuristic principle we follow is to take only the points which are not too close together. This leads us to use the heuristic rule of taking the next candidate point $x^{*}$ which minimises the sum of the inverse cubic distances to the already selected points, i.e. $1 /\left\|x_{1}-x^{*}\right\|^{3}+\ldots+1 /\left\|x_{c}-x^{*}\right\|^{3}$. Furthermore, two versions of this approach are considered, referred to as update strategy 0 and update strategy 1 in the paper. The former is more greedy in a sense, it keeps track of the (smaller) training set, and on each proxy update event it extends the training set by adding a few recruits from the new candidates. The latter strategy keeps track of the (bigger) candidate set, and on each proxy update event, it selects the training set from scratch from all candidates. More details on these are given in Appendix A, sub-section proxy training scheme. Although the proper design of computer experiments is beyond the scope of this work, there exists a generous research on this topic, which is lightly overviewed in the same place in Appendix A.

Using the surrogate models to aid the evaluation of expensive likelihood in the MCMC methods has been a topic of ongoing research. The approaches proposed in the literature include (to give a few examples):
- Engineering-flavoured workflows exposing a multitude of tools, but lacking theoretical rigour, e.g. [8, 26].

- Biased samplers, where the estimator's bias results from the use of the surrogate model. Some approaches tend to avoid considering this bias, while focusing on the other aspects of the workflow, e.g. [7] who consider a nonstationary GP response surface applied to a PDE-based simulator, and [39] who treat a big data problem with the stochastic gradient technique adopted from the machine learning. The other approaches make attempt to estimate and handle the bias, see e.g. [23] tackling a big data problem, and [10] who try to make the bias vanish asymptotically.

- Unbiased samplers, which usually rely on a surrogate model when making a proposal step, but calculating the exact likelihood on the Metropolis-Hastings accept-reject step, thus maintaining the zero bias. One of the examples is the surrogate transition method, see Liu [27], p. 194, where a proposal step of a sampler is made up of multiple steps performed by a cheap proxy model, followed by an accept-reject step involving the original (expensive) target. Such approach is natural for the samplers that exhibit random walk behaviour, e.g. RWM or MetropolisAdjusted Langevin Algorithm (MALA) [15]. But for the Hamiltonian Monte Carlo, where the proposals usually consist of multiple steps of the integrator, a more natural unbiased procedure would be to perform the integration based on the proxy model, and make an acceptreject decision based on the actual target. This approach was followed, e.g. in [24, 33], and it is employed in our work as well.

The papers cited usually deal with problems of small dimension, hardly exceeding 10 . This limitation may stem either from the need to avoid unnecessary complexity in the numerical illustrations (in this case, the algorithms should be in principle easily applicable to higher dimensions), or it may indicate more fundamental challenges in applying the proxy models to aid MCMC (which seems to be the case at least for the procedure considered in our work).

Our paper has two major goals:

1) To provide a neat exposition of the method from the formulas and complete algorithmic details to the theoretical grounds of the unbiased sampler.

2) To perform a wide range of experimental tests, targeting the questions:

- What are the benefits of proxy + HMC over the RWM?

- Is it worth using the gradients for the proxy training, in our context?

- What are the feasible problem dimensions, and when does the considered approach start to break down? 
- How do the two proxy approaches (simple proxy vs. data proxy) compare? In fact, this comparison continues one of the directions of further work outlined in [24].

The rest of the paper is organised as follows. First, we briefly describe the Bayesian formulation of the inverse problem (Section 2), overview the core algorithmic part of the MCMC samplers (Section 3), the adjustments performed during the burn-in stage (Section 4) and discuss the concept of the effective sample size (Section 5). Then, three case studies are presented in Section 6: a toy problem with Rosenbrock function, a small synthetic reservoir simulation model Punq-S3, and a synthetic single-phase reservoir simulation model with 40 wells. The main part of the paper is concluded with the discussion of the results in Section 7. The complementary detailed Appendix A describes the construction of the kriging proxy model (the equations, the derivatives, the hyperparameters adaptation, training on values and on gradients, additional training schemes, simple proxy and data proxy approaches). The approach to handling the domain boundaries in HMC is described in Appendix B, and the proxy model settings used in different numerical examples - in Appendix C.

\section{Problem set-up, Bayesian formulation}

We consider a problem of uncertainty quantification for a simulation model describing a physical system (e.g. reservoir simulation), by means of generating a set of samples from the posterior distribution. Taking a Bayesian approach, the target posterior distribution density $\pi\left(x \mid d_{o}\right)$ for model $x$ given the observed data $d_{o}$ is defined as the product of the prior density for model $x$ and the likelihood function $\mathcal{L}\left(x \mid d_{o}\right)$. In this work, for simplicity of the experimental part, and without loss of generality we only consider uniform priors over the parameter space, and the latter is defined as a region with simple bounds $l_{i} \leq x_{i} \leq u_{i}$. This also means that the target posterior probability density function (pdf) is proportional to the likelihood, restricted to the parameters region. In the rest of the paper, we will also use the term objective function which is defined as

$$
\begin{aligned}
f(x)= & -2 \cdot \log \mathcal{L}\left(x \mid d_{o}\right) \equiv \\
& \left(d(x)-d_{o}\right)^{T} C^{-1}\left(d(x)-d_{o}\right),
\end{aligned}
$$

where $d(x)$ is the simulator output for model $x$, arranged in a vector form; $d_{o}$, observed data; and $C$, covariance matrix for the data errors, which will be diagonal throughout this work. As can be seen, the formula uses the Gaussian likelihood model. For a diagonal $C$, the objective function $f(x)$ is a weighted sum of squares (1), which is usually minimised during history matching, and hence the name objective function.

\section{RWM and HMC samplers}

We use two Markov chain Monte Carlo algorithms for sampling from the target distribution $\pi(x):^{3}$

1. Random walk Metropolis (RWM) with Gaussian proposals, which is a basic reference sampler.

2. Hamiltonian Monte Carlo (HMC), which is a more advanced sampler relying on the gradients of the log likelihood.

RWM Random walk Metropolis is a simple sampler which makes local proposals based on Gaussian distribution $N\left(0, C_{p}\right)$, and Metropolis-Hastings accept-reject steps with probability of accepting transition from $x$ to $y$ equal to

$a(x \rightarrow y)=\min \left(1, \frac{\pi(y)}{\pi(x)}\right)$

Note that the proposal density is not used in the formula, since it is zero-centered Gaussian, and hence symmetric. In our experiments, the covariance matrix $C_{p}$ used in the proposal step is taken equal to $\varepsilon^{2} I$, where the step size $\varepsilon$ is tuned to achieve certain target acceptance rate for the chain.

HMC The idea of Hamiltonian Monte Carlo is based on complementing the space of the main variable $x \in \mathbb{R}^{d}$ (which is treated as coordinate vector) by the momentum variable $p \in \mathbb{R}^{d}$, and simulating the Hamiltonian dynamics for $\{x, p\}$, which allows making non-local proposals, see Neal [29], Girolami and Calderhead [15], Lan, Bui-Thanh [24], Bou-Rabee and Sanz-Serna [6].

Treating $L(x) \equiv-\log \pi(x)$ as potential energy, and $\frac{1}{2} p^{T} M^{-1} p$ as kinetic energy, the full energy will be given by Hamiltonian $H(x, p)=L(x)+\frac{1}{2} p^{T} M^{-1} p$. It is easy to see that $\exp (-H(x, p))$ is up to a normalising factor the joint pdf for $\{x, p\}$, where $x$ has the target distribution $\pi(x)$, momentum has a Gaussian distribution $p \sim N(0, M), x$ and $p$ are independent, and $M$ is a constant mass matrix. HMC algorithm generates samples from the joint distribution, and then only takes vectors $x$, which have the marginal distribution equal to the target distribution.

Given the current state of the Markov chain $\left\{x_{i}, p_{i}\right\}$, the new proposal is generated in three steps. At the first step, momentum randomisation, a random momentum vector is drawn, $p_{*} \sim N(0, M)$. At the second step, Hamiltonian dynamics move, the new coordinate and momentum are found by evolving the Hamiltonian flow over some interval $[0, T]$ of fictitious time $t$, starting from $\left\{x_{i}, p_{*}\right\}$, following the equations

\footnotetext{
${ }^{3}$ For the sake of notation simplicity, in what follows we drop the conditioning of the target distribution on $d_{o}$, assuming the observed data to be fixed.
} 


$$
\begin{aligned}
& \frac{d x}{d t}=\frac{\partial H}{\partial p}=M^{-1} p \\
& \frac{d p}{d t}=-\frac{\partial H}{\partial x}=-\nabla x L(x)
\end{aligned}
$$

In most cases, integrating these equations is not feasible in the closed form, and integration is done numerically. A common approach is to use the explicit leap frog (Stormer-Verlet) integrator, discretising the time interval $[0, T]$ with steps of size $\varepsilon$ :

$$
\begin{gathered}
p(t+\varepsilon / 2)=p(t)-\varepsilon \nabla_{x} L(x(t)) / 2 \\
x(t+\varepsilon)=x(t)+\varepsilon M^{-1} p(t+\varepsilon / 2) \\
p(t+\varepsilon)=p(t+\varepsilon / 2)-\varepsilon \nabla_{x} L(x(t+\varepsilon)) / 2
\end{gathered}
$$

This integrator, mapping $\left\{x_{i}, p_{*}\right\} \rightarrow\left\{x^{*}, p^{*}\right\}$, is timereversible and volume-preserving, so it can be used as a part of the Metropolized Reversible Map algorithm [6], with the accept-reject step properly formulated. This leads us to the third step within the HMC proposal, Metropolis-Hastings accept-reject, accepting the new point $\left\{x^{*}, p^{*}\right\}$ with probability

$$
\begin{aligned}
& a\left(\left\{x_{i}, p_{i}\right\} \rightarrow\left\{x^{*}, p^{*}\right\}\right) \\
& \quad=\min \left(1, \exp \left(-H\left(x^{*}, p^{*}\right)+H\left(x_{i}, p_{*}\right)\right)\right)
\end{aligned}
$$

If the exact integration of Eq. (4) was available, it would preserve the full energy $H(x, p)$, leading to all proposals being accepted. The numerical leapfrog integrator does not preserve $H(x, p)$; however, the integration error is rather small, of the order $O\left(\varepsilon^{2}\right)$ for a fixed time interval $[0, T]$, so the acceptance rate in the third step will typically be high, even if the proposal steps traverse considerable distances in the parameter space.

When performing integration of the Hamiltonian dynamics, we adopt a simple approach, fixing the number or leapfrog steps to a value typically from 10 to 50 , and adjusting the step size $\varepsilon$ so as to reach the acceptance rate of 0.6-0.9 [4]. If the leapfrog trajectory happens to be too long, the final point may, following a quasi-elliptical orbit, return close to the starting position, thus wasting computational effort on short moves. To circumvent this, methods like No-U-Turn Sampler (NUTS) have been proposed by Hoffman and Gelman [20]. However, in all our calculations, occurrence of the U-turn trajectories was negligible, so no additional technique to handle them was used.

The HMC procedure described so far is only applicable to the target distributions with the gradients of the density readily available. Next, we turn to the HMC application for a target distribution $\pi_{0}(x)$ where we do not have efficient code to calculate the derivatives of $L_{0}(x)=-\log \left(\pi_{0}(x)\right)$. Although the target $L_{0}(x)$ lacks derivatives, it can used to calculate the Hamiltonian $H_{0}(x, p)=L_{0}(x)+\frac{1}{2} p^{T} M^{-1} p$. For efficient estimation of the gradients used by the HMC proposal step, a proxy model ${ }^{4} L(x)$ is built, $L(x) \approx L_{0}(x)$. The corresponding approximate target $\pi(x)=\exp (-L(x))$ is now easy to sample from if one applies the HMC procedure described; however, this is not our goal.

Instead, we want HMC to sample from the target $\pi_{0}(x)$, but performing the Hamiltonian dynamics move (4), (5) on the $L(x)$ and its gradients. To ensure that the Markov chain obtains the correct stationary distribution $\propto \exp \left(-H_{0}(x, p)\right)$, the acceptance probability should be changed from (6) to the formula involving the target Hamiltonian $H_{0}(x)$ :

$$
\begin{aligned}
& a\left(\left\{x_{i}, p_{i}\right\} \rightarrow\left\{x^{*}, p^{*}\right\}\right) \\
& \quad=\min \left(1, \exp \left(-H_{0}\left(x^{*}, p^{*}\right)+H_{0}\left(x_{i}, p_{*}\right)\right)\right)
\end{aligned}
$$

Once again, we are in the situation of the Metropolized Reversible Map algorithm outlined in [6] (Algorithm 5.1 and the relevant proposition), and hence, the proxy-aided HMC will indeed sample from the target $\pi_{0}(x)$, although internally it relies on the proxy gradients ${ }^{5}$. Note that each evaluation of (7) requires one calculation of the target likelihood at the proposed point, i.e. a simulator run. The results of this simulator run can then be reused for the subsequent proxy training.

The energy conservation properties of the Hamiltonian flow integrator normally result in high acceptance probabilities in (6); however, the acceptance probability (7) may suffer if the proxy model happens to lack precision compared to the target (the simulator). This may render the proxy-aided HMC inefficient, and such situations will be illustrated in the numerical examples in Section 6.

\section{Burn-in stage of the MCMC}

Before the Markov chain has reached the stationarity stage, it passes through the initial transient burn-in sampling. The burn-in samples are discarded afterwards, and are not used in any uncertainty quantification. This allows making different kinds of on-the-fly adjustments to the sampling algorithm during the burn-in stage, without having to worry about the rigorous aspects of the MCMC method (like the detailed balance).

We apply two types of such adjustments, both of them are made periodically, i.e. every $n_{K}$ iterations of the MCMC

\footnotetext{
${ }^{4}$ Although the proxy model is introduced here as a new entity, for convenience we reuse the previous notation: $L(x), \pi(x), H(x)$ - these letters without subscript now refer to the proxy.

${ }^{5}$ In notation of the said reference, the Hamiltonian flow based on the proxy model provides the volume-preserving map $\Phi$ which is reversible with respect to the linear involution $S$ (the latter simply flips the momentum sign), and hence, the joint distribution of $\{x, p\}$ is invariant under the Hamiltonian dynamics move kernel.
} 
sampler during the burn-in stage (the typical values we use as $n_{K}$ are 10-50). The first adjustment is tuning (decreasing/increasing) the step size $\varepsilon$ used by the sampler proposal move, so as to achieve a predefined acceptance rate. The tuning is made based on comparison of the target acceptance rate and the actual acceptance rate calculated for the last $n_{K}$ samples. If the actual acceptance rate is too small, $\varepsilon$ is decreased, and vice versa.

The second adjustment is update of the proxy model used by the HMC sampler, by adding some of the points visited by the sampler to the proxy design points set. More details on the proxy update procedure can be found in Appendix A.

We note that we do not attempt to rigorously estimate the actual duration of the Markov chain burn-in. Instead, based on the observed mixing of the chain, and to ensure reasonable computational times 3000-5000 iterations were typically taken in the burn-in stage. From the algorithmic point of view, this number delimits the initial stage where the two mentioned adjustments take place.

\section{Effective sample size (ESS)}

Any Markov chain Monte Carlo method generates a sequence of samples which are, in general, correlated. The presence of the correlations increases the convergence error of MCMC estimates compared to the ideal case of non-correlated samples [6].

More specifically, for the non-correlated i.i.d. random variables $x_{i}$ with distribution $P(\cdot)$, a quantity of interest $\mu \equiv \int g(x) d P(x)$ can be approximated by the sample mean estimator $\widetilde{\mu}_{n}=\frac{1}{n} \sum_{i=1}^{n} g\left(x_{i}\right)$, and following the Central Limit Theorem, the Monte Carlo error for the estimator $\widetilde{\mu}_{n}$ is defined as $\sigma_{0} / \sqrt{n}$, where $\sigma_{0}^{2}$ is the variance of (any of) $g\left(x_{i}\right)$.

Likewise, for a reversible, irreducible Markov chain $\left\{x_{i}\right\}$ with stationary distribution $P(\cdot)$, the quantity of interest $\mu \equiv \int g(x) d P(x)$ can be approximated by the MCMC estimator $\widetilde{\mu}_{n}=\frac{1}{n} \sum_{i=1}^{n} g\left(x_{i}\right)$, and following the appropriate Central Limit Theorem, the MCMC error of $\widetilde{\mu}_{n}$ is defined as $\sigma / \sqrt{n}$, where $\sigma^{2}$ is the asymptotic variance of $\sqrt{n} \widetilde{\mu}_{n}$, satisfying

$\sigma^{2}=\sigma_{0}^{2}+2 \sum_{i>1} \operatorname{Cov}\left(g\left(x_{1}\right), g\left(x_{i}\right)\right)$,

see $[6,13]$ for more details.

There exist a number of practical estimators for the asymptotic variance, and in our work, we adopt the initial monotone sequence estimator [13]. This estimator essentially uses (8), replacing the lagged autocovariances by the empirical autocovariances, and truncating the summation at appropriate step.

Rather than reporting the asymptotic variance it is more convenient to use the concept of effective sample size
(ESS), denoted $n_{\text {eff. }}$ This quantity says how many hypothetical i.i.d. samples do we need to reach the same Monte Carlo error as the real MCMC error we have for $n$ samples. From this definition, $\sigma / \sqrt{n}=\sigma_{0} / \sqrt{n_{\text {eff }}}$. Here, $\sigma_{0}^{2}$ is the usual variance of (any of) $g\left(x_{i}\right)$, which is in practice estimated by the sample variance formula applied to the Markov chain. In other words, it is the autocovariance at lag zero (also cf. (8)). Thus, when dealing with the samples (series) generated by an MCMC algorithm, reporting the ESS is an easy, although not exhaustive, way to show the quality of the series. If the samples are highly correlated, the ESS will be small, up to tens and units. For the series with little correlations, the ESS will approach the full sample size $n$.

We note however that the ESS cannot be used as a criterion that the Markov chain has converged to the target stationary distribution, and can be used for making confident estimates. E.g., there may be situations with high ESS values, but the chain failing to explore the parameter space properly. An example of such behaviour is illustrated bellow in the 5D Rosenbrock problem, sub-case (c), where the ESS is the biggest, but the histograms show rather large errors.

\section{Case studies}

\subsection{D Rosenbrock}

The first numerical example to illustrate the use of proxy models in MCMC takes a simple objective function equal to the 5D Rosenbrock function, further defining the likelihood via (2). The objective function in question is given by formula (9), and the parameter space is restricted to the region $[-2,2]^{5}$.

$f(\vec{x})=\sum_{i=1}^{4} 100\left(x_{i+1}-x_{i}^{2}\right)^{2}+\left(1-x_{i}\right)^{2}$

We considered a simple proxy with a small number of training points in the initial design, and more emphasis on training during the Markov chain burn-in. The proxy settings can be found in Appendix C. Apart from these, some other settings of the sampler and the proxy were varied, resulting in six cases a-f displayed in Fig. 1 and Table 1. These cases are:

- Proxy is trained only using the target function values (cases a, b, c, f), or both the function values and the gradients (cases d, e).

- Using the update strategy 0 (cases a, d), or update strategy 1 (cases b, c, e, f). See Appendix A for details on the update strategies 0 and 1 .

- Burn-in: either short burn-in of 500 samples, or long burnin of 3300 samples (the latter is case $\mathrm{f}$ ). 


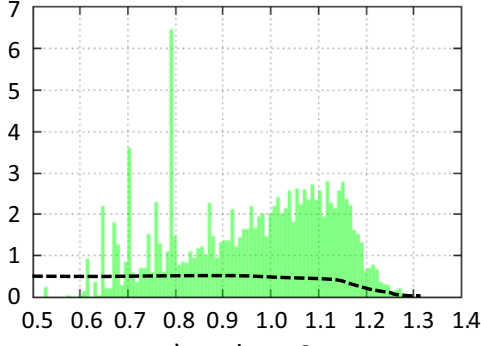

a) update-0

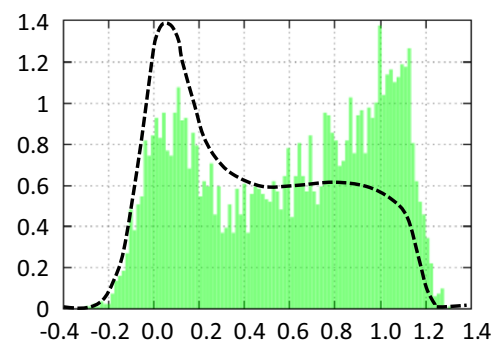

d) update-0, with gradients

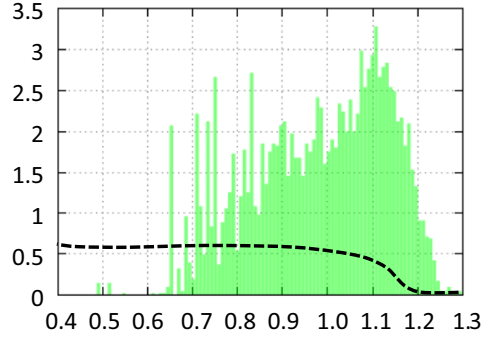

b) update-1

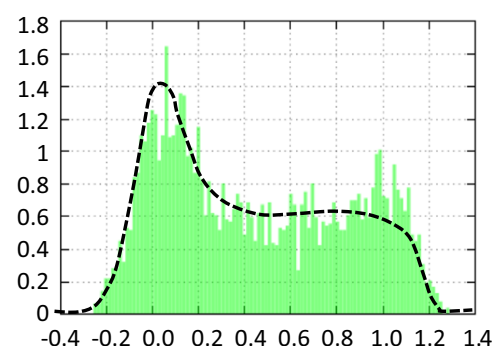

e) update-1, with gradients

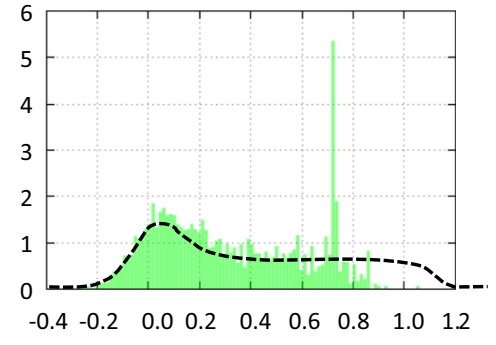

c) update-1, more points

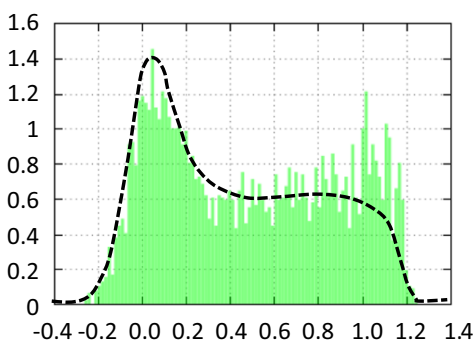

f) update-1, long B/I

Fig. 1 Histograms for component $x_{3}$ of the 5D vector sampled by HMC-with-proxy for the 5D Rosenbrock example. The black dashed lines show the exact histogram profiles (for reference). For the other components $x_{i}$, the picture is qualitatively the same

- Case c, where increased number of design points is taken, initial design points: 35 points from Sobol sequence +1 starting point, and additional design points taken during burn-in: 6 points for every 10 iterations.

In all cases, the proxy is used only to get the gradients for the leap frog integration in HMC sampler. In this integration, 10 leap frog steps were used, with the step size $\varepsilon$ tuned during burn-in so as to achieve the target acceptance rate of 0.7. The HMC sampler employed the unity mass matrix. Five thousand samples were generated for all the considered proxy set-ups a$\mathrm{f}$, and based on them, the histograms were produced, as shown in Fig. 1.

Analysing the sampling results given by the parameters histograms, and the effective sample sizes, the following can be noted. The HMC with a proxy trained only on function values during short burn-in, has essentially failed (cases $a, b$ ).

If the proxy is trained with the gradients, it gets much more information on the underlying function. This can be seen e.g. in the increase of the effective rank of kriging matrix from 62 (cases a, b) to 332, 342 (cases d, e). Adding the gradients slows down the entire sampling (in this case, by factor 2 2.5); however, the HMC is now capable of sampling from the target distribution with reasonable accuracy. We also note that even though case e does not perfectly reproduce the reference histogram contours, the errors present here are comparable to the errors of $\mathrm{HMC}$ sampling from the exact 5D Rosenbrock without the use of proxy (not shown on the plots), so these errors are due to somewhat insufficient sample size.

Comparing the proxy update strategies 0 and 1, i.e. cases a, $d$ vs. cases b, e, improvements for update strategy

Table 1 Performance and effective sample size for the HMC-with-proxy sampling for the 5D Rosenbrock example. The rows correspond to the different proxy settings a-f

\begin{tabular}{|c|c|c|c|c|c|c|c|c|c|}
\hline \multirow[t]{2}{*}{ Case } & \multicolumn{2}{|c|}{ Used in proxy training } & \multirow[t]{2}{*}{ Eff. rank of krig. matrix } & \multicolumn{5}{|c|}{ Effective sample size } & \multirow[t]{2}{*}{ CPU, sec } \\
\hline & Func. vals & Gradients & & $x_{1}$ & $x_{2}$ & $x_{3}$ & $x_{4}$ & $x_{5}$ & \\
\hline a) update- 0 & 56 & 0 & 62 & 144 & 57 & 38 & 34 & 36 & 19.9 \\
\hline b) update-1 & 56 & 0 & 62 & 342 & 81 & 57 & 51 & 51 & 24.5 \\
\hline d) update- 0 , with gradients & 56 & 56 & 332 & 45 & 47 & 46 & 36 & 48 & 49.5 \\
\hline e) update-1, with gradients & 56 & 56 & 342 & 258 & 277 & 212 & 183 & 181 & 55.9 \\
\hline c) update- 1 , more points & 366 & 0 & 342 & 347 & 415 & 358 & 265 & 507 & 115.9 \\
\hline f) update-1, long B/1 & 366 & 0 & 342 & 194 & 270 & 240 & 220 & 214 & 2938.0 \\
\hline
\end{tabular}


1 can be seen in the histograms, effective ranks of kriging matrix, and the effective sample sizes. This goes at the price of the increased computation time. However, for case e (as compared to case d), the ESS increase is 4-5 times, and the computation time is only $13 \%$ higher, which indicates the efficiency of the proxy update strategy 1 for this example.

As discussed, adding the information form the gradients during proxy training proved beneficial for the proxy accuracy and applicability for HMC. One may then ask-what if the same amount of information is supplied to the proxy by means of the increased number of the function values, instead of using the gradients. This option was tested in cases $\mathrm{c}$ and $\mathrm{f}$, which have the same rank of kriging matrix as the gradients case e. Simply increasing the number of design points on every proxy update event by factor of 6 (case c) resulted in poor sampling. A different approach which gave plausible results (case f), used the non-increased number of design points on every update event, but increased duration of the burn-in stage, where the additional proxy training takes place: 3300 instead of 500 samples. However, despite good histograms and effective sample sizes, case $\mathrm{f}$ showed prohibitively long sampling time. So, for the considered 5D Rosenbrock example the use of gradients proved to be more efficient than supplying the "same amount" of information by the function values alone.

\subsection{PunqS3 three-phase model}

As an example of a 3D three-phase reservoir simulation model, a well-known Punq-S3 model was considered [1, 12], its dataset in the Eclipse simulator format can be found on the website. The model grid has dimensions $19 \times 28 \times 5$ cells with unknown porosities and permeabilities. The reservoir has a small gas cap, and underlying water, with a Carter-Tracy aquifer strong enough to support the reservoir pressure.

There are 6 production wells perforated in the oil zone, for which a production period of 8 years is considered. For this period, the following observed well data are available: bottom-hole pressures, gas-oil ratios, and the water cuts. These data, along with the standard deviations can be found on the same website. However, the original website data also contain the increased "weights" for some data points, which we did not use in the formulation of the likelihood.

We employed the same model parameterisation as was introduced by Hajizadeh [18], and Abdollahzadeh, Reynolds [2]. It involves defining 45 regions, i.e. 9 regions in each of the 5 layers, see Fig. 2 (left), and specifying a constant porosity in each of these regions; thus, the model parameters are 45 porosity values.

Then, given the porosity $\varphi$, horizontal and vertical permeabilities are calculated by the formulas: $k_{h}=\exp (0.77+9.03 \varphi)$

$k_{v}=3.124+0.306 k_{h}$

The ranges for parameters corresponding to the different layers are a bit wider than in Hajizadeh [18], and are listed in Fig. 2 (right).

A heuristic verification that the choice of the likelihood and parameterisation is reasonable, can be done by considering the values of the objective function after a minimisation run. If the forward model was linear, then the objective function at the minimum would have a $\chi^{2}$ distribution with $N_{d}-N_{p}$ degrees of freedom, where $N_{d}$ is the number of data points, and $N_{p}$, the number of parameters, see discussion in appendix 6.8 in [38], and paragraph 8.1.1 in [31]. For the realistic nonlinear models, this criterion is not applicable rigorously, but can still serve as a guideline. In our exercise with Punq-S3 the successful minimisation runs resulted in the minimum objective function values of 65-75, which is in a good agreement with the above criterion, provided that we have 117 data points and 45 model parameters, so the expected minimum value of the objective function should be around $N_{d}-N_{p}=72$.

When performing MCMC sampling runs, we considered different dimensions of the parameter space. To do that, an optimisation run was first performed, giving a sub-optimally history-matched model. For the history-matched model, some of the 45 parameters were then fixed, while others remained active parameters. The MCMC sampling was performed in the space formed by these active parameters. The smallest considered parameter space dimension was 6 , with 6 active parameters corresponding to the regions where the six well were perforated. The other considered space dimensions were $11,16,21$ etc.

For each case of parameter space dimension, two samplers were tested: random walk Metropolis (RWM) and Hamiltonian Monte Carlo (HMC). Both had a burn-in stage of 5000 samples and the main stage of 30,000 samples. For the RWM sampler the step size $\varepsilon$ was adjusted during burn-in to keep the acceptance rate of 0.2 .

For the HMC sampler, 20 steps were used in the leapfrog integration, and the step size $\varepsilon$ was tuned to get the proxylevel $^{6}$ acceptance rate of 0.7 . The mass matrix was set equal to the unity matrix. Since the derivatives from the reservoir simulator are not available, using the gradients from the proxy model is the only way to run HMC. The settings used in defining the proxy model can be found in Appendix C.

To illustrate the MCMC results, Fig. 3 shows the parameter trace (value vs. iteration number) and the final histogram for parameter $\varphi_{21}$. Porosity region 21 corresponding to this parameter is located in 3 rd layer, and contains perforation of well

\footnotetext{
${ }^{6}$ This means the acceptance rate based on (6), which only accounts for the leapfrog integration errors, and is rather high. The final (simulator-level) acceptance rate is based on (7), it in addition accounts for the proxy errors, and is typically lower.
} 
Fig. 2 Left: parameterisation regions for the Punq-S3 model. Nine regions for the top layer are shown. The other layers have the same layout. Right: ranges for the parameters in different layers

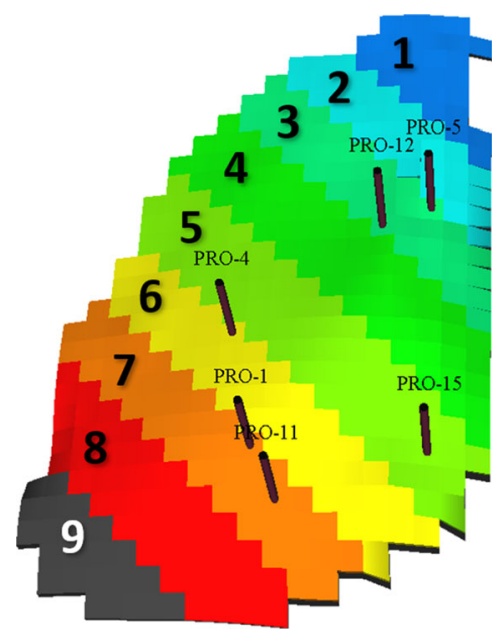

\begin{tabular}{|c|c|c|}
\hline layer & $\boldsymbol{\varphi} \min$ & $\boldsymbol{\varphi} \max$ \\
\hline 1 & 0.07 & 0.35 \\
\hline 2 & 0.05 & 0.15 \\
\hline 3 & 0.07 & 0.35 \\
\hline 4 & 0.07 & 0.3 \\
\hline 5 & 0.07 & 0.35 \\
\hline
\end{tabular}

PRO-5. Since the cases of the different parameter space dimension, strictly speaking, correspond to the different inverse problems, the resulting histograms across the different rows are not expected to be the same, although they look quite similar. Also, note that in any of the rows corresponding to dimensions $6,11,16$, the histograms for RWM as compared to HMC would be the same, if the RWM sampler was run for a sufficiently long time.

The running time for RWM and HMC samplers is reported in Table 2. For HMC, the average time per sample during the burn-in stage is rather high because of many proxy training events, and if proxy update strategy 0 was selected instead of strategy 1 , this time would be lower. However, even for strategy 1 , the average time per sample for the entire run is dominated by the simulator CPU time, and not by the proxy housekeeping, so the HMC costs (5.4 s/sample) are only slightly higher than the RWM costs ( $4.5 \mathrm{~s} / \mathrm{sample})$.

The results for the RWM sampler show lack of exploration of the parameter space, very low effective sample size (ESS), and very slow chain mixing, as can be seen on the parameter trace plots. This means that the RWM-generated samples are highly correlated, and the estimates made from them would not have sufficient confidence. Opposite to this, results from the HMC procedure for dimensions $6,11,16$ show a good mixing and high ESS reaching thousands and tens of thousands. This allows making reasonably reliable estimates from the HMC-generated samples, e.g. calculating the means, quantiles, histograms, etc. Thus, for the low-dimensional problems, HMC is a feasible MCMC sampling technique for the considered reservoir simulation problem, whereas RWM is not.

As the parameter space dimension increases to 21, 26, 31, HMC performance notably degrades, and the sampler occasionally gets stuck in a single point for thousands of iterations. This happens because of the insufficient accuracy of proxy occurring for the parameter spaces of higher dimensionality. The proxy accuracy degradation occurred even for lower dimensions of 6-16, as evidenced by the gradually decreasing ESS for these cases. For dimensions of 21 and higher, up to the full dimension of 45 (plots not shown here), HMC results essentially become useless.

Behaviour of the proxy accuracy vs. problem dimension is also illustrated in Table 3, where we can observe the error monotonically increasing, and this increase has rather exponential nature. To calculate the proxy error shown in the second column of the table, we first considered the quantities involved in the accept-reject probabilities (6)-(7), and for each generated model sample calculated the error value $e=\frac{1}{2}\left|f_{0}\left(x_{i}\right)-f_{0}\left(x^{*}\right)-\left(f\left(x_{i}\right)-f\left(x^{*}\right)\right)\right|$, where $f_{0}$ is the objective function for the simulator, $f$, objective function for the proxy model, $x_{i}$, point before the proposal move, $x^{*}$, the new proposed point. The second column in Table 3 displays the median of all $e$ 's over the 30,000 samples. The quantity $e$ is basically the difference between the logarithms of the acceptance probabilities on the proxy level (6), and on the simulator level (7). We are ultimately interested in the acceptance probability on the simulator level, which will be essentially decreased by $\exp (e)$ compared to the typically high acceptance probability on the proxy level. When the error $e$ becomes greater than 1 for dimensions 21 and higher, the degradation of the acceptance probability becomes especially notable, resulting in too many rejections, as was observed in the examples of dimension 21,26,31. This analysis also shows that the HMC algorithm poses very stringent requirements on the proxy accuracy: the objective function error (proxy vs. simulator) should not exceed 1.0-2.0 for the algorithm to work. This tolerance for the objective function error is small indeed, for example, compared to the value of objective function itself. During MCMC the latter would be approximately equal to the number of observed data points $N_{d}$ (at least for linear problems). So, for each data point $i$ the average discrepancy of simulator vs. observation is allowed to be $\approx \sigma_{i}$, whereas the average discrepancy of simulator vs. proxy should be 
$\frac{\varepsilon}{\bar{\sigma}}$

6
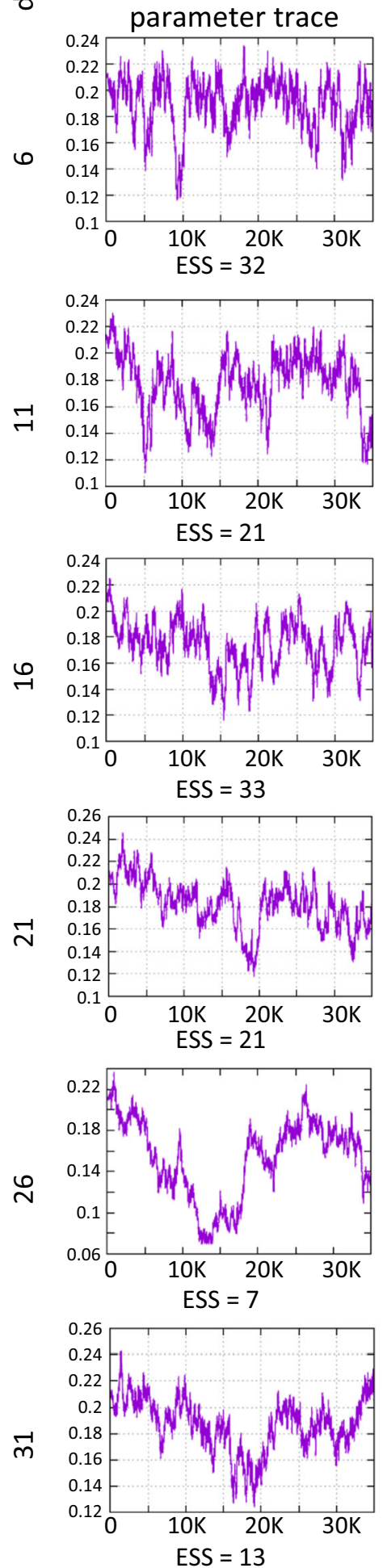

Fig. 3 Traces and histograms for parameter $\varphi_{21}$ for different dimensionality of the parameter space (shown in rows). For the trace plots (in columns 1,3 ), $x$-axis shows the iteration number, $y$-axis shows

\section{HMC}
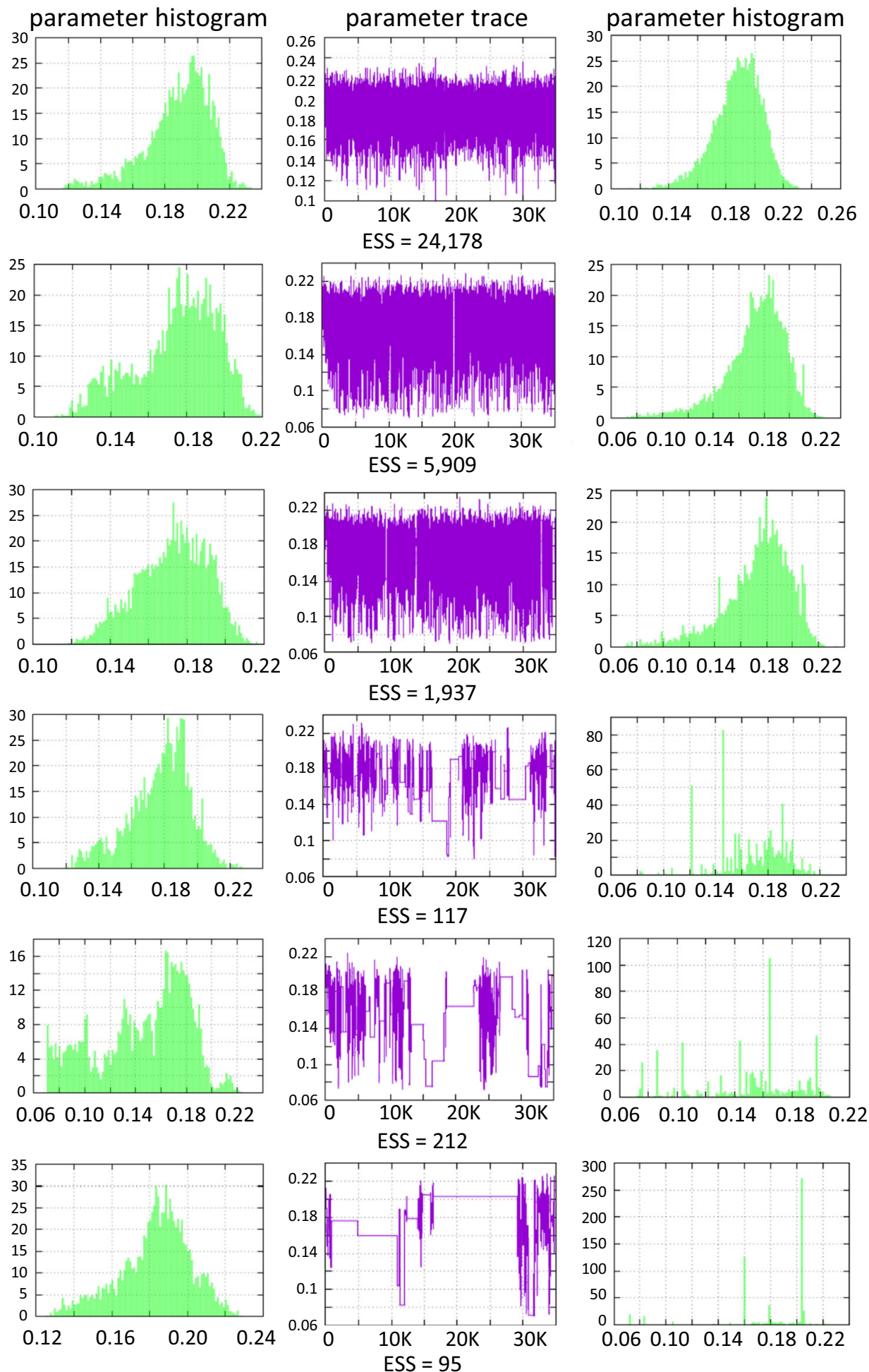

the parameter value. For the histogram plots (in columns 2, 4), $x$-axis shows the parameter value. Effective sample size for $\varphi_{21}$ for each case is also reported 
Table 2 MCMC running time for RWM and HMC, Punq-S3 case. The numbers shown here essentially do not depend on the dimensionality $(6$, 11,16 etc)

\begin{tabular}{llll}
\hline Sampler & \multicolumn{2}{l}{ Time for stage, hours } & \\
& Burn-in & Main & Total \\
RWM & 6.4 & 37.0 & 43.3 \\
HMC & 12.9 & 39.8 & 52.7 \\
& \multicolumn{4}{l}{ Avg time per sample, seconds } \\
Sampler & Burn-in & Main & \\
& 4.6 & 4.4 & Total \\
RWM & 9.3 & 4.8 & 4.5 \\
HMC & \multicolumn{2}{c}{} \\
\hline
\end{tabular}

$\approx \sigma_{i} / \sqrt{N_{d}}$ per data point, where $\sigma_{i}$ is the standard deviation used in the objective function definition.

Certain increase of the proxy accuracy could be achieved by increasing the number of design points. However with the number of points reaching a few thousand the proxy calculations would notably slow down, but the dimensionality of the parameter space feasible for the proxy-based HMC would only increase insignificantly.

To conclude this section, we briefly illustrate the advantages of data proxy vs. simple proxy. Firstly, consider an example of how HMC performance is affected when one uses either type of proxy. Figure 4 shows the parameter trace plots for the two sampler runs, both of them used the problem of dimension 16, and identical settings, the only difference being the type of the proxy model: simple proxy or data proxy. As can be seen, the latter results in a notably better behaviour, wider parameter space exploration and much faster chain mixing, which can be attributed to higher accuracy of the data proxy.

Then, we compare the accuracy of the two proxy types directly, by plotting the objective function as a function of two parameters $\varphi_{33}$ and $\varphi_{42}$, with the other parameters being fixed at the near-optimum values (Fig. 5). The output from the simulator, as well as from the two proxy models, is shown. For both proxies, the training set consisted of 1600 Sobol points in 16-dimensional space, plus the near-optimum point. This design is different from what was used in the MCMC runs,

Table 3 Proxy errors for the main sampling stage of HMC. Rows correspond to the different problem dimensionality. The second column shows the median value of $e$ (see the main text above for the definition of $e$ )

\begin{tabular}{rl}
\hline Dim & Proxy error \\
\hline 6 & 0.054 \\
11 & 0.51 \\
16 & 0.76 \\
21 & 8.4 \\
26 & 9.4 \\
31 & 13.4 \\
\hline
\end{tabular}

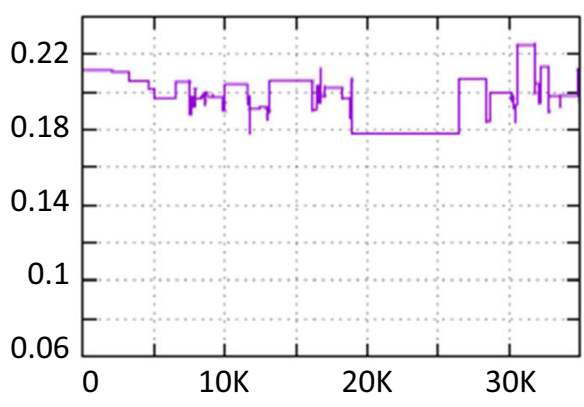

HMC with simple proxy $\operatorname{dim}=16$

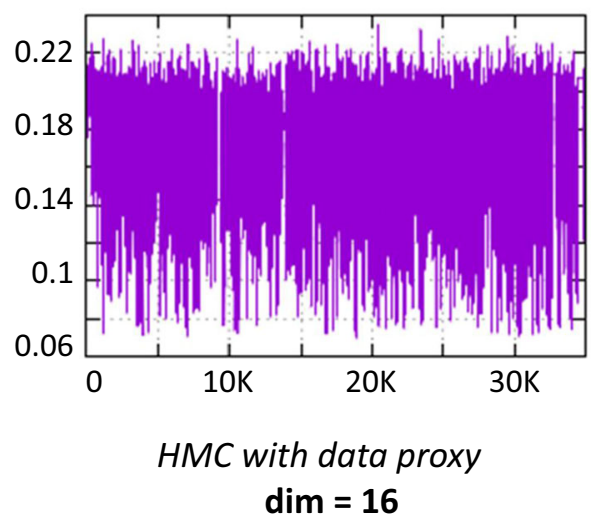

Fig. 4 Comparison of the $\varphi_{21}$ parameter trace for HMC with simple proxy (top) and HMC with data proxy (bottom, same as in Fig. 3). Problem dimension $=16$

although it has the same number of points. The plots indicate that in the area of interest where the objective function values are small, the data proxy is notably more accurate in reproducing the exact objective function than the simple proxy (in fact, the data proxy is one order of magnitude more accurate).

\subsection{Single-phase simulation for a synthetic petroleum reservoir}

The third case study considers the forward modelling performed by a single-phase simulator of a subsurface reservoir. The simulator works on an unstructured grid using a finite volume discretisation, calculating the dynamics of pressures and fluid flows. The reservoir properties are described by the pore volumes $V_{i}$ of the blocks, transmissibilities $T_{i j}$ between the interconnected blocks, and perforation productivity indices $J_{k}$ for the well-block connections. In the forward simulation, the reservoir properties are fixed, the wells-producers and injectors - are controlled by the flow rates, and the output modelled data are the time-dependent pressures in the blocks and the bottom-hole pressures (BHP) at wells.

When solving the inverse problem, the observed block pressures and BHP data are known for certain wells and time steps, and the reservoir properties $V_{i}, T_{i j}, J_{k}$ are to be resolved. 

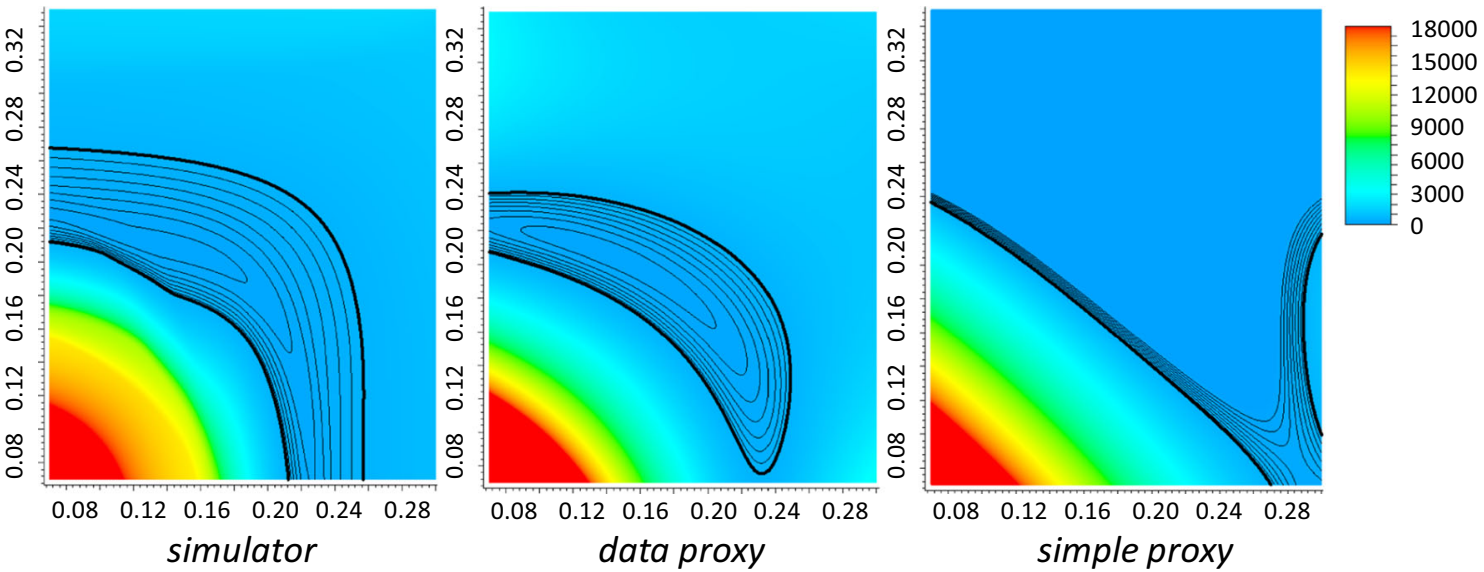

Fig. 5 Plot of the objective function (coloured map) vs. $\varphi_{33}$ (x-axis) and $\varphi_{42}$ (y-axis). The contours have the step 50, and highlight the region with low objective function values (100 to 500). Left to right: output from the simulator, the data proxy, and the simple proxy

The objective function (2), as well as the likelihood, are calculated taking the simulator-modelled data and the observed data. The simulator we are using also allows calculating the gradients of the objective function by the adjoint procedure, so that HMC sampler can be run directly, without the use of a proxy model.

In this case study, although the simulator and the spatial discretisation are rather simple, we considered a synthetic reservoir of a more realistic size: is has 7 layers, and 40 producing/injecting wells. The whole simulation is for a 12 year time period.

The grid used in the single-phase simulation consists of large blocks, roughly speaking in each layer we take only one block per well, plus some occasional additional blocks, plus some blocks on the boundary to represent the active aquifers. The perforations of all 40 wells in the seven layers $(A-G)$ are shown in Fig. 6. As can be seen, most of the wells are perforated in the two main layers $A$ and E, where we may therefore expect better resolution of the reservoir parameters. In the remaining five layers with little wells, higher uncertainties are anticipated.

To synthesize the observed pressure data, reservoir simulation was performed on a fine-grid precursor model. The precursor model (it was actually created in the first place) is not only using a different grid discretisation, but also slightly different physics: it is a three-phase (oil, water, gas) model, run by the black oil Schlumberger Eclipse simulator. The resulting output Eclipse pressures were perturbed by the uncorrelated Gaussian noise, with $\sigma=$ 20 bar for the well BHP data, and $\sigma=3$ bar for the reservoir (block) pressure data. Altogether, 380 measurement points were considered (365 for BHP, 15 for reservoir pressure), taken with time step of 6 months. Here, we note that by using one modelling procedure (Eclipse) to get the observed data, and a different procedure (singlephase simulator with a coarse grid) to invert for the reservoir parameters, we are not in a situation of an inverse crime [22].

The preliminary tests with the MCMC samplers revealed that if we take the full range of the available parameters $V_{i}, T_{i j}, J_{k}$, which means 338 unknown parameters altogether, only HMC with the gradients directly from the simulator is capable of sampling in a satisfactory way. The resulting effective sample sizes for the parameters (as a fraction of the total sample size) are quite high, ranging from 0.02 to 0.88 , with the average value equal to 0.35 . However, this sampling is rather slow, generating 61 samples per hour, due to the numerous runs of the simulator even for a single proposal move.

Building an accurate proxy for such high dimensionality is hardly feasible. In the tests conducted, the proxy-based HMC failed, getting stuck in a single point, with the average simulator-proxy error equal to 170 . To make the proxy work,

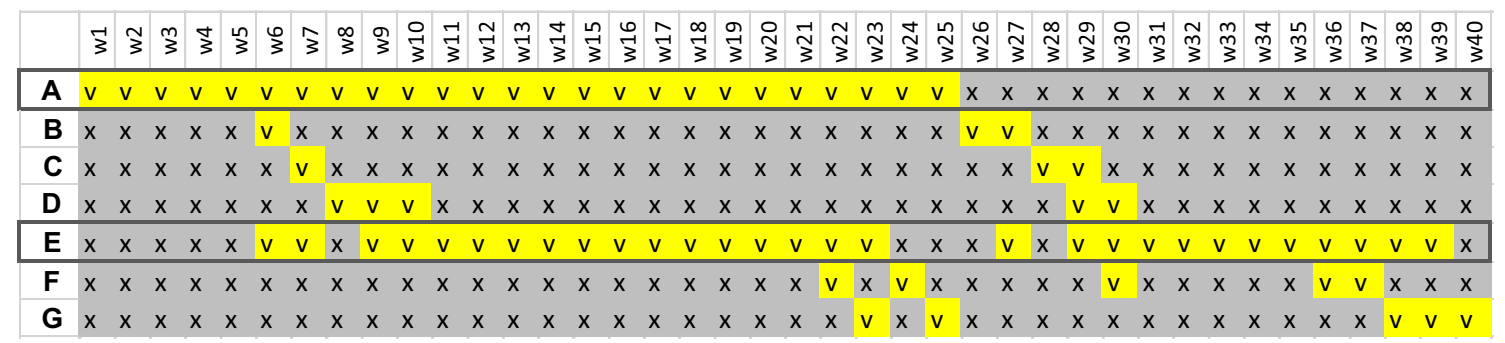

Fig. 6 Perforations of the 40 wells in the seven layers. Yellow " $v$ " means that a particular well (column) has perforation in the given layer (row) 
a simplified problem was considered with the number of parameters reduced to 21 . In this lightweight parameterisation, we employed three parameters per layer: one pore volume multiplier for the aquifer blocks, one transmissibility multiplier, and one perforation productivity multiplier. To find the base case to which these 21 multipliers should be applied, an optimisation (history matching) run was performed with the full range of 338 unknowns. An example of the well BHP behaviour from the maximum likelihood (history-matched) model is shown in Fig. 7. Then, all the non-aquifer pore volumes were fixed, since they normally have rather small prior uncertainty. For the aquifer pore volumes, as well as the transmissibilities and productivity coefficients, the respective multipliers were applied. All the 21 multipliers were set to vary from 0.1 to 10 .

In what follows, we present the results of running the two MCMC samplers: RWM and HMC with unity mass matrix.
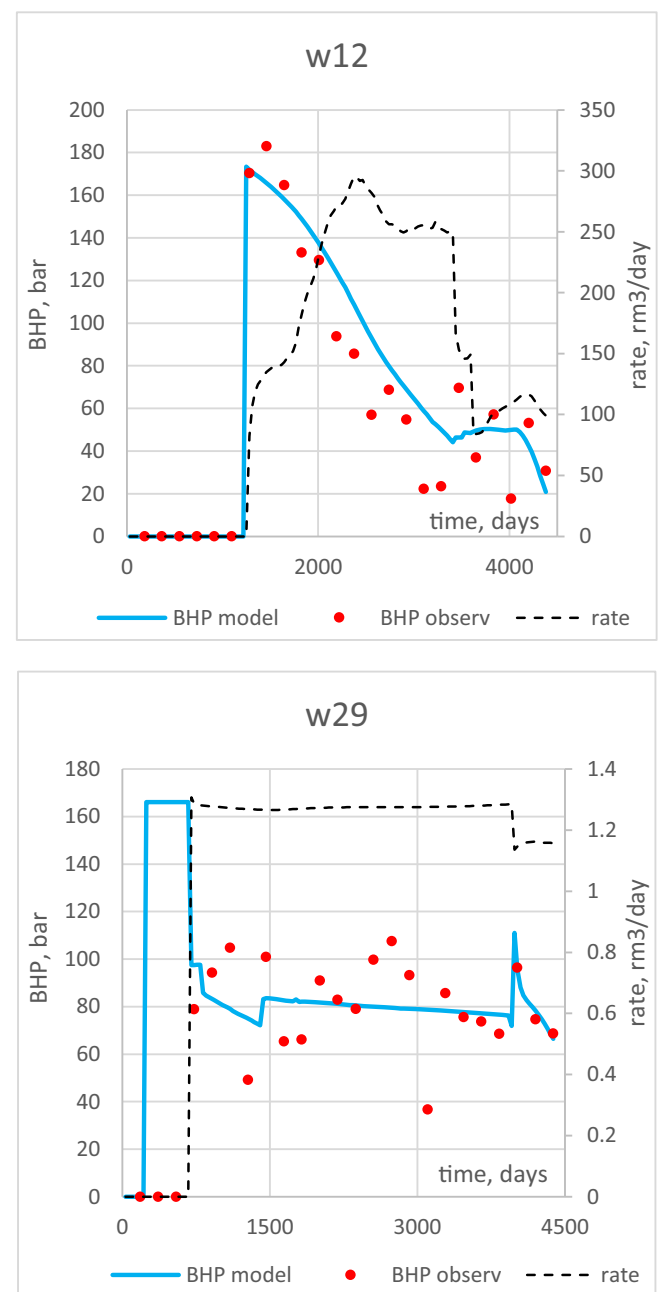

Fig. 7 Well rates and bottom-hole pressures for wells w12 and w29. Dashed black line, volumetric flow rate at reservoir conditions; solid blue line, BHP from the history-matched base case model (output from the single-phase simulator); red dots, synthetic observed BHP data (output from Eclipse plus Gaussian noise)
For RWM, the target acceptance rate was set to 0.2 , and for HMC, the target proxy-level acceptance rate was set to 0.8 . These target rates are reached by the sampler by automatically tuning the step size $\varepsilon$ during the burn-in stage. For HMC, 50 leap frog steps were used to generate each proposal. Besides, for the HMC sampler we considered five sub-cases:

- HMC using the gradients of the objective function provided directly by the simulator.

- HMC using a simple proxy to get the gradients needed by the algorithm. Since the accurate gradients are available from the simulator, the main motivation for adopting the proxy gradients is to save the computation time.

- HMC using a simple proxy trained by both the objective function values and the simulator-provided gradients. The gradients used for the proxy training are only taken in a small number of design points, whereas the gradients output by the proxy model are used extensively in all leap frog integrations. When a proxy model is trained with the gradient information, the kriging matrix will grow $d+1=$ 22 times faster. To prevent the kriging matrix size from excessive growing, the number of design points was decreased by factor of 20 .

- HMC using a data proxy with update strategy 1.

- HMC using a data proxy with update strategy 0 (faster). Since the number of the modelled data points 380 is quite large, it makes sense to run the data proxy calculations in a parallel mode. For both cases with the data proxy, we employed 3 parallel processes for handling the data proxy computation.

More details on the proxy model parameters can be found in Appendix C. All the samplers were run with 3000 samples in the burn-in stage, and 50,000 samples in the main stage.

Running the different $\mathrm{MCMC}$ cases revealed that using the simple proxy with HMC does not work - apparently because of too high proxy errors. This was the case both for training the simple proxy with the function values, and for training it with the function values and gradients.

Figure 8 shows the trace for one of the model parameters, for both approaches. It can be seen that the Markov chain starts exploring the parameter space, gradually increasing the step size, to reach the target acceptance rate 0.8 on the proxy level. At some point when the step size is large enough, the proxy accuracy becomes too low, the accept-reject step starts rejecting all the proposals, and the sampler gets stuck in a single point.

The other samplers did not experience such severe problems as the HMC with simple proxy, and their running time is reported in Table 4. Although RWM has the lowest running time, it fails to explore the parameter space properly (Fig. 9), and thus is not a suitable choice of the MCMC sampler. 

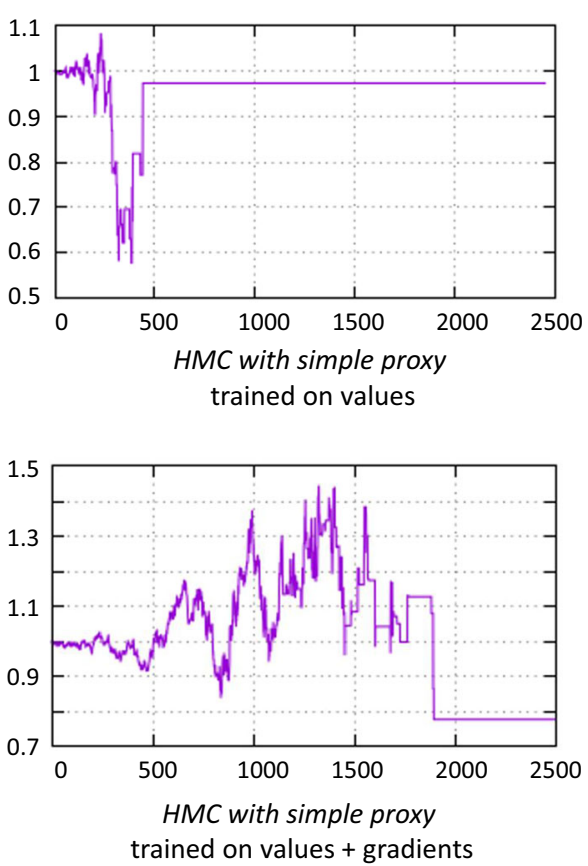

Fig. 8 Trace for the first parameter (aquifer volume multiplier for layer A). Top, HMC with simple proxy trained on values; bottom, HMC with simple proxy trained on values + gradients. $X$-axis shows the iteration number, $y$-axis parameter value

Figure 9 displays the posterior histograms for the first two model parameters: aquifer volume multipliers for layers A and B. Three samplers are shown: RWM, HMC with the simulator-provided gradients, and HMC with data proxy and update strategy 0 . The poor exploration for RWM is especially visible on the "layer B" plot, where RWM only explores a small part of the actual range. The two HMC samplers show consistent results, with HMC + data proxy case having more noisy histograms, which is natural due to the smaller effective sample sizes compared to the case of HMC + exact gradients. We also note that the posterior uncertainty for the "layer B" parameter is higher than for "layer A", this can be explained by layer A having much more wells perforated in it, and hence more observed data.

Although HMC with the exact gradients resulted in the Markov chain with the highest effective sample sizes, its running time is an order of magnitude bigger than for the proxyaided samplers. For a fair comparison of the three HMC samplers, Fig. 10 shows the ESS per hour for the 21 parameters. As can be seen, for most parameters the proxy-aided versions of HMC have higher efficiency (higher ESS/hour) than the HMC with exact gradients, and, among the two proxy-aided HMC samplers, the one with update strategy 0 performs better than the strategy-1 version (a behaviour opposite to what was observed for the 5D Rosenbrock example).

Overall, the considered case study has shown that even when the simulator is capable of providing the gradients of the objective function for the HMC sampler, using the proxy model can accelerate the HMC and improve its efficiency.

\section{Discussion}

In this work, we studied the application of proxy models to aid the Markov chain Monte Carlo sampling for the Bayesian inverse problems. We described the underlying principles for building a kriging proxy mod$\mathrm{el}$, and training the proxy model based on the target function values (and optionally, the gradients). Two proxy types were considered and compared: proxy for the log likelihood (simple proxy), and proxy for the simulator-output data (data proxy). Both of them allow easy calculation of the gradients and higher order derivatives, which can be used in the advanced MCMC samplers (and in the optimisation algorithms, not considered in this work). Two simple heuristic strategies for training the proxy model during the initial MCMC stage were proposed. Employing more elaborated methods for the design of computer experiments is one of the natural directions for the future work. It should be noted that the simple proxy (single GP) and the data proxy (a quadratic combination of many GPs) would require different approaches for the adaptive design of computer experiments.

The kriging proxy formulation allows adapting/optimising the kriging hyperparameters, which is reported to certain degree in the literature [3, 24]. However, in our experiments, such an adaptation (implemented for three parameters: nugget $\mathrm{n}$, correlation radius $r$, and Matérn parameter $\nu$ ) did not result in the proxy quality improvement, and was left for the future work. Although the results of these experiments are not reported in our paper, the methodology of the hyperparameters adaptation is outlined in Appendix A for reference. It is anticipated that this approach may work better for the situation of a wider set of the hyperparameters, e.g. in the case of
Table 4 MCMC running time for the synthetic 40-wells model

\begin{tabular}{lll}
\hline Sampler & RWM & HMC, exact grads \\
Run time, hours & 13.4 & 693.4 \\
\hline Sampler & HMC, data proxy, update-1 & HMC, data proxy, update-0 \\
Run time, hours & 73.2 & 49.5 \\
\hline
\end{tabular}




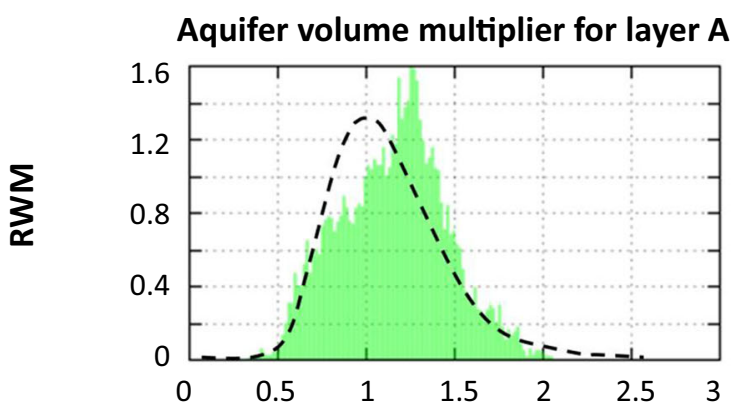

\section{Aquifer volume multiplier for layer B}
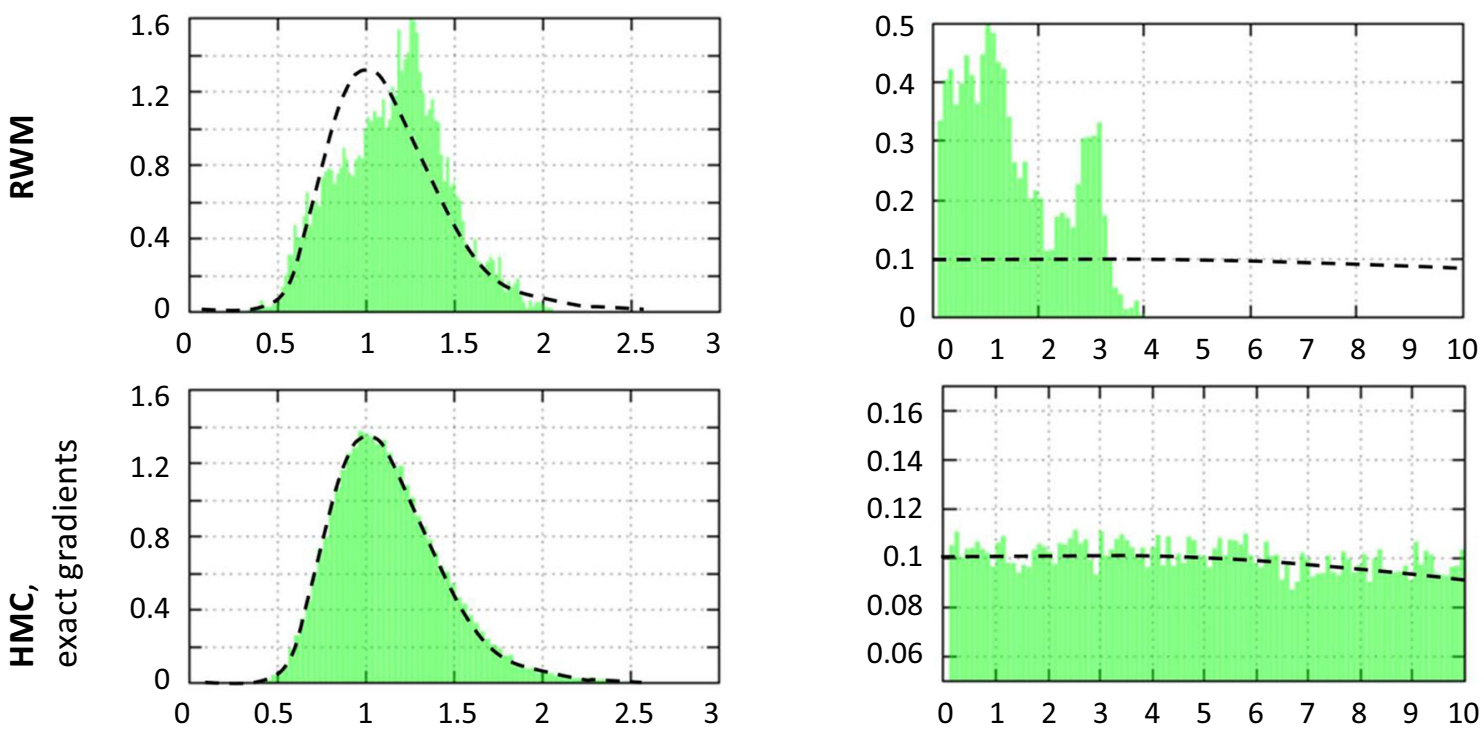

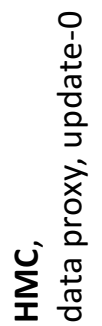

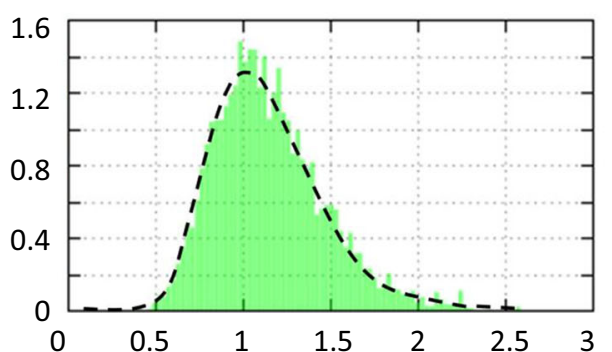

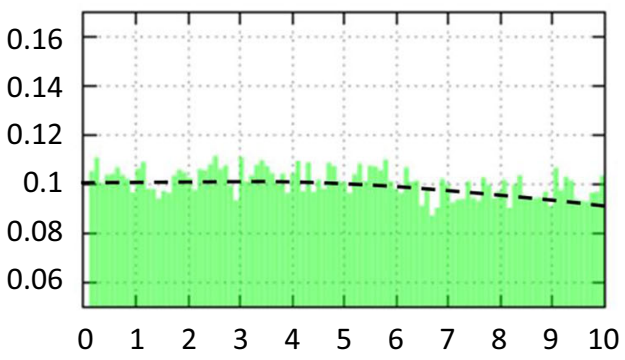

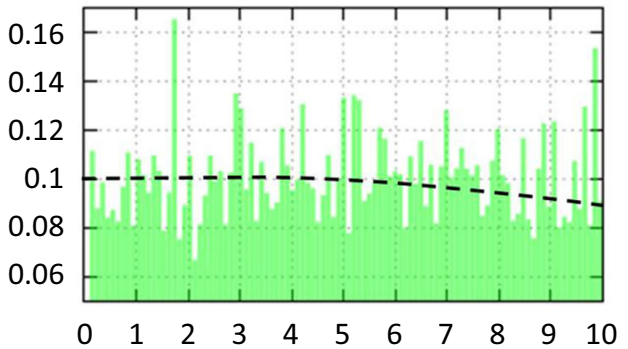

Fig. 9 Posterior histograms for the two model parameters (shown in two columns), resulting from the different samplers (shown in three rows). $X$-axis displays the parameter values. The dashed black outline, shown here for reference, was drawn manually based on the results from the HMC with exact gradients

anisotropic covariance, with different correlation radii in different directions, which are to be optimised. Handling the anisotropic covariance should be addressed in the future developments. Another direction which is worth further consideration is employing multi-fidelity kriging to surrogate both the high fidelity response (e.g. from the base-case simulation

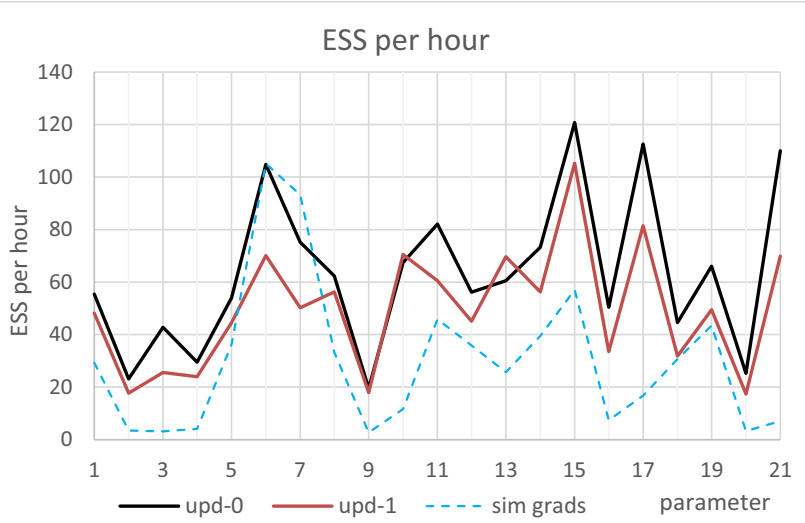

Fig. 10 Effective samples size per hour ( $y$-axis) for the 21 parameters $(x$ axis), HMC sampling. The lines correspond to the cases: data proxy with update strategy 0 (black), data proxy with update strategy 1 (red), and simulator-provided gradients (blue dashed) model) and low fidelity response (e.g. from the coarsened simulation model).

The efficiency of an HMC-like sampler may be increased if it accounts for the curvature of the landscape of the target density. This can be done e.g. by taking the mass matrix equal to the Fisher Information (FI) matrix. If the mass matrix is allowed to vary throughout the parameter space, the HMC procedure should be adjusted in order to produce reversible proposals, e.g. as done by Riemannian HMC algorithm (RHMC). Or, still accounting for the geometrical information, a slightly different proposal scheme can be adopted, like Manifold-based Metropolis-Adjusted Langevin Algorithm (MMALA). Such algorithms require the calculation of the FI matrix and its derivatives with respect to the model parameters, which is rarely available from the simulators. Like with the gradients, one can employ a proxy model to calculate an approximate FI matrix. The MCMC approaches using the curvature information are known in the literature $[15,24,25,28]$, but are beyond the scope of this paper.

In order to apply the proxy model in the MCMC sampling, we considered a modified unbiased HMC 
sampler, where the gradient-friendly proxy model is used to make the proposal steps, and the exact simulation model is used to make the accept-reject decisions. This approach allows generating the samples from the actual target distribution.

To test the efficiency of the proxy-aided MCMC, three case studies were examined, including two reservoir simulation models. The following was observed:

- The basic RWM sampler has very poor performance for the reservoir simulation problems, and can hardly be used in practice.

- Proxy-aided HMC is a feasible MCMC sampler for the problems of small dimension (20 parameters or less, pretty much problem dependent). The proxy's job is either to provide the derivatives for the HMC where such derivatives are not available from the simulator; or if the simulator can also output the necessary derivatives, the proxy model can be used to provide the approximate derivatives at a smaller computational cost.

- Data proxy works notably better than the simple proxy.

- For small problems, training a proxy model with the gradient values (provided the gradients are output by the simulator) may be beneficial. However, only simple proxy allows such an approach, which is quite restrictive. The other restriction is the fast growth of the kriging matrix size, limiting the feasible number of the design points. From our experience, using the data proxy, albeit without the training with gradients, is more efficient.

- Comparing the two simple heuristic schemes for additional proxy training during the Markov chain burn-in, either of them may be more efficient than the other, depending on the problem.

One of the major bottlenecks of the proxy-aided MCMC scheme is the stringent requirements on the proxy accuracy for the sampler to work. This results in the limited dimensionality of the problems which can be tackled. To push those limits, some radical improvement of the proxy accuracy is required. While further increase of the number of the design points is not an option due to the kriging matrix size issue, an alternative which is worth considering is a continuous proxy update during the entire MCMC sampling, taking aboard the new points and removing the old ones. However, straightforward application of this approach with the classical HMC is not possible because the proposal move becomes irreversible once the proxy model update is allowed. So, a careful re-design of the underlying sampler would be needed.

Acknowledgements This work would not have been possible without sponsorship of the EQUIP (Enabling Uncertainty Quantification for Inverse Problems) grant from the Engineering and Physical Sciences
Research Council (EPSRC) EP/K034154/1. We also thank Schlumberger for providing the Eclipse simulator to Heriot-Watt University, and acknowledge the use of the open source software libraries OpenMPI, BLAS, LAPACK, ALGLIB.

\section{Appendix A. Kriging proxy models}

The proxy models we consider in this paper are the emulators based on Gaussian processes (GP), or kriging. The details of GP approach can be found, e.g. in Sacks, Welch [35], Lan, Bui-Thanh [24], Oakley [30]. We briefly describe the main concepts.

\section{Kriging proxy via Gaussian process}

Consider a function $g: \mathbb{R}^{d} \rightarrow \mathbb{R}$, and $n$ design points $x_{1}, \ldots, x_{n}$ in $\mathbb{R}^{d}$. Denote by $Y_{s}$ the $n \times 1$ vector of values of $g(\cdot)$ at the design points. Based on this data, a kriging proxy function $G: \mathbb{R}^{d .} \rightarrow \mathbb{R}$ can be constructed, which is smooth enough, and reproduces either exactly or approximately the values of $g(\cdot)$ at the design points.

To build the proxy, consider a stationary Gaussian process $\boldsymbol{Y}(x)=\sum_{j=1}^{T} \beta_{j} f_{j}(x)+Z(x)$, where the mean is defined by $T$ trend functions $f_{j}: \mathbb{R}^{d} \rightarrow \mathbb{R}$ selected in advance, and $T$ coefficients $\beta_{j}$ which do not depend on $x \in \mathbb{R}^{d}$. The simple choices of the trend functions considered in this work include, for example, a constant trend $f_{0} \equiv$ const, and linear trend functions $f_{k}(x)=x^{(k)}, k=1, \ldots, d$. The stochastic part of $\boldsymbol{Y}(x)$ is given by the stationary Gaussian process $Z(x)$ with zero mean. We consider $Z(x)$ to have isotropic covariance

$\operatorname{Cov}(Z(x), Z(y))=\sigma^{2} c\left(\frac{\|x-y\|}{r}\right)$,

where $\sigma$ is the standard deviation, $r$ correlation radius, and $c(\cdot)$ is a one-dimensional covariance function, with $c(0)=1$.

In our work, we employ two kinds of 1D covariance functions: Gaussian, $c(\tau)=(1-\mathrm{n}) \exp \left(-3 \tau^{2}\right)$; and Matérn, $c(\tau)=(1-\mathrm{n}) \frac{2^{1-\nu}}{\Gamma(\nu)}(\sqrt{12 \nu} \cdot \tau)^{\nu} K_{\nu}(\sqrt{12 \nu} \cdot \tau)$, for $\tau>0$, where $0 \leq \mathrm{n}<1$ is a nugget, $\Gamma(\cdot)$ gamma function, $K_{\nu}(\cdot)$ modified Bessel function of the second kind.

We introduce the following notation of vectors and matrices involved in description of the Gaussian process at the design points: $Y_{n \times 1}$ the vector of values of the Gaussian process $\boldsymbol{Y}(\cdot)$ at the design points; $F_{n \times T}$ values of the trend functions $f_{j}(\cdot), j=1, \ldots T$, at the design points; $\beta_{T \times 1}$ vector of the trend coefficients, for which we consider a weak prior distribution over $\mathbb{R}^{T} ; R_{n \times n}=\frac{1}{\sigma^{2}} \operatorname{Cov}(Y, Y)$ correlation matrix for the GP at the design points, with $R_{i, j}=c\left(\left\|x_{i}-x_{j}\right\| / r\right)$. Here, the subscripts of the form $p \times q$ denote the matrix sizes, they will be omitted later. Given this notation, the conditional 
distribution of vector $Y \mid \beta$ is Gaussian $N\left(F \beta, \sigma^{2} R\right)$ with pdf

$$
(2 \pi)^{-\frac{n}{2}}\left|\sigma^{2} R\right|^{-\frac{1}{2}} \exp \left\{-\frac{(Y-F \beta)^{T} R^{-1}(Y-F \beta)}{2 \sigma^{2}}\right\},
$$

where $|\cdot|$ denotes determinant of a matrix. Given the value of the random vector $Y$ is equal to $Y_{s}$, one can find the maximum likelihood estimate (MLE) of coefficients $\beta$ by maximizing the pdf (12): $\widehat{\beta}=\left(F^{T} R^{-1} F\right)^{-1} F^{T} R^{-1} Y_{s}$. Furthermore, it can be shown that $\beta \mid Y=Y_{S}$ has Gaussian distribution $N\left(\widehat{\beta}, \sigma^{2}\left(F^{T} R^{-1} F\right)^{-1}\right)$.

Next, take an arbitrary point $x \in \mathbb{R}^{d}$ where the proxy estimate is to be made, denote $y(x)$ the value of the Gaussian process $\boldsymbol{Y}(\cdot)$ at $x$; denote $f(x)_{T \times 1}$ the vector of values of the trend functions at $x$; and denote by $r(x)_{n \times 1}=\frac{1}{\sigma^{2}} \mathrm{Cov}$ $(y(x), Y)^{T}$ the vector of correlations between GP at $x$ and GP at the design points, with $r(x)_{i}=c\left(\left\|x-x_{i}\right\| / r\right)$. The Gaussian process $\boldsymbol{Y}(\cdot)$ at the extended set of points $\left\{x, x_{1}\right.$, $\left.\ldots, x_{n}\right\}$ has values $\left(y(x), Y^{T}\right)^{T}$, which follow a multivariate Normal distribution. Finally, the value of kriging proxy is provided by the mean of the conditional distribution of $y(x)$ given $Y=Y_{s}$ :

$$
\begin{aligned}
G(x) & \equiv \mathbb{E}\left(y(x) \mid Y=Y_{s}\right) \\
& =f^{T}(x) \widehat{\beta}+r^{T}(x) R^{-1}\left(Y_{s}-F \widehat{\beta}\right)
\end{aligned}
$$

In this formula, the value of $\beta$ was taken equal to $\widehat{\beta}$, so one can think of it as if the MLE estimate of $\beta$ was used. Or, the same expression (13) can be obtained if one first considers the posterior joint distribution of $\{y(x), \beta\}$ given $Y=Y_{s}$, and integrates out $\beta$, see Oakley [30] for details. Taking this second route, one would get a Gaussian distribution for $y(x)$ given $Y=Y_{s}$, with mean equal to the right hand side of (13), and variance equal to

$$
\begin{aligned}
& \sigma^{2}\left[1-r^{T} R^{-1} r+\right. \\
& \left.\quad\left(f^{T}-r^{T} R^{-1} F\right)\left(F^{T} R^{-1} F\right)^{-1}\left(f^{T}-r^{T} R^{-1} F\right)^{T}\right] .
\end{aligned}
$$

Note that the value of standard deviation $\sigma$ does not affect the kriging estimate $G(x)$, but affects the kriging error (14).

\section{Kriging proxy via MSE minimisation}

Another way to obtain the kriging estimate (13) is sketched as follows. As before, we consider a random process $y(x)$ with mean value equal to $f^{T}(x) \beta$ at point $x$, and covariance structure defined by the right hand side of (11).

Consider an estimate given by the random variable $\widehat{y}(x)=\lambda^{T}(x) Y=\sum_{i=1}^{n} \lambda_{i}(x) Y_{i}$, which involves the position- dependent deterministic kriging coefficients $\lambda_{i}(x)$, and values $Y_{i}$ of the random process at the design points. To get the kriging estimate, one needs to make $\widehat{y}(x)$ to be unbiased estimate of $y(x)$, i.e. require $\mathbb{E} \widehat{y}(x) \equiv \lambda^{T} F \beta=f^{T}(x) \beta \equiv \mathbb{E} y(x)$ for all $\beta$. Next, minimise the mean squared error (MSE), which equals $\mathbb{E}[\hat{y}(x)-y(x)]^{2}=\operatorname{Var}[\hat{y}(x)-y(x)]$, conditioned to the unbiasedness. Omitting the details, the solution to this problem boils down to a system of linear equations from which the vector $\lambda(x)$ can be found. Finally, taking $Y=Y_{s}$, the kriging estimate is obtained as $G(x)=\lambda^{T}(x) Y_{s}$, which can be shown to be identical with (13). Expression (13) can also be re-written in the blockmatrix form:

$G(x)=\left(\begin{array}{ll}r^{T} & f^{T}\end{array}\right)\left(\begin{array}{cc}R & F \\ F^{T} & 0\end{array}\right)^{-1}\left(\begin{array}{c}Y_{S} \\ 0\end{array}\right)$

The corresponding MSE is then equal to (14), and it can also be re-written in the block-matrix form as

$\sigma^{2}\left[1-\left(\begin{array}{ll}r^{T} & f^{T}\end{array}\right)\left(\begin{array}{cc}R & F \\ F^{T} & 0\end{array}\right)^{-1}\left(\begin{array}{l}r \\ f\end{array}\right)\right]$

The matrix which is being inverted in (15) and (16) is referred to as the kriging matrix in our work.

\section{Behaviour at the design points and smoothness of the kriging proxy}

If nugget $\mathrm{n}$ is equal to zero in the definition of the 1D covariance function $c(\cdot)$, then it can be shown that the kriging proxy $G(x)$ is exact at the design points, i.e. it exactly reproduces the underlying function $g(\cdot)$. This is easy to demonstrate because for $x=x_{i}$, we have $\left(r^{T}(x) f^{T}(x)\right)$ is equal to the $\mathrm{i}$-th row of the kriging matrix, so the right hand side of (15) will give the i-th component of $Y_{s}$, which is the exact value of $g(\cdot)$ at the design point $x_{i}$.

If $\mathrm{n}>0$, then $G(x)$ at the design points is exact, but has a discontinuity. This can be seen e.g. in the simplified case of one design point, and no trend functions. In this case the kriging estimate is $G(x)=c\left(\left\|x-x_{1}\right\| / r\right) \cdot g\left(x_{1}\right)$, so it essentially reproduces $c(\cdot)$ radially from the point $x_{1}$.

To get rid of the discontinuity, one needs to apply 1D covariance with $c(0)=1-\mathrm{n}$ when calculating the vector $r(x)$, but keep $c(0)=1$ when calculating the correlation matrix $R$. This trick only affects kriging behaviour at the design points, making the kriging proxy continuous and inexact at them.

The need to use a non-zero nugget may arise, e.g. if function $g(x)$ is an output of a simulator which uses iterative algorithms for calculations. Such a simulator is likely to have some noise due to the rounding errors and iterative nature of the solver, and these errors may show discontinuous behaviour in response to the minor 
perturbations of the simulator inputs. There is little point to reproduce the noisy simulator output exactly by the kriging proxy. Another motivation for using a non-zero nugget is to avoid singularity in the kriging matrix. However, in practice, rank deficiency of the kriging matrix is not a critical issue since this matrix can be inverted in a generalised sense, e.g. using procedure DGELSS from LAPACK library. We also note that in the case of rank deficiency, the kriging proxy may not be exact at the design points.

In the case of Matérn 1D covariance $c(\cdot)$, the corresponding Gaussian process will be $\lceil\nu\rceil-1$ times mean square differentiable, and the corresponding kriging proxy $G(x)$ will be $2(\ulcorner\nu\rceil-1)$ times differentiable [37], where $\lceil\cdot\urcorner$ denotes the ceiling function. For the Gaussian 1D covariance, which is the limit case of Matern for $\nu \rightarrow \infty$, the kriging proxy is infinitely differentiable.

\section{Derivatives of the kriging proxy}

For use with the MCMC samplers which employ gradients and metric tensors, we will need the first and the second derivatives of the kriging proxy. A straightforward way to obtain the gradient of $G(x)$ is to differentiate the expression (13) with respect to $x$. The only items depending on $x$ are $f^{T}(x)$ and $r^{T}(x)$. So,

$\nabla G(x)=d f^{T}(x) \widehat{\beta}+d r^{T}(x) R^{-1}\left(Y_{s}-F \widehat{\beta}\right)$

where matrix $d f^{T}(x)$ has $\mathrm{i}$-th column equal to $\nabla f_{i}(x), i=1, \ldots T$, and matrix $d r^{T}(x)$ has i-th column equal to $c^{\prime}\left(\left\|x-x_{i}\right\| / r\right) /$ $\left(r\left\|x-x_{i}\right\|\right) \cdot\left\{x-x_{i}\right\}, i=1, \ldots n$. In a similar way one can find the second derivatives of $G(x)$, but we omit these details.

\section{Adapting the kriging hyperparameters}

During derivation of kriging proxy, we found the maximum likelihood estimate $\beta=\widehat{\beta}$ for $Y=Y_{s}$ and fixed $\sigma^{2}, R$, by maximising expression (12) with respect to $\beta$. In the same way, one can estimate the MLE of $\sigma^{2}$ for fixed $\widehat{\beta}$ and $R$ to be $\widehat{\sigma}^{2}=\frac{1}{n}\left(Y_{s}^{T} R^{-1} Y_{s}-\widehat{\beta}^{T} F^{T} R^{-1} Y_{s}\right)$. Furthermore, MLE can be found for the kriging hyperparameters controlling the correlation matrix $R$. In the simplest case such hyperparameters would include the nugget $\mathrm{n}$, correlation radius $r$, and the smoothness parameter of the Matérn covariance function $\nu$, denoted together by a vector $\xi=\{\mathrm{n}, r, \nu\}$. In a more advanced setting, one could consider anisotropic covariance instead of (11), and kriging hyperparameters vector $\xi$ would include correlation radii for different directions.

When maximising (12) with respect to $\beta, \sigma^{2}, \xi$, for each $\xi$ we can find the optimum $\beta=\widehat{\beta}(\xi)$ and $\sigma^{2}=\widehat{\sigma}^{2}(\xi)$. Plugging the latter two into (12), we maximise it with respect to $\xi$. This problem is equivalent to minimising $h(\xi)=\widehat{\sigma}^{2}(\xi) \cdot(\operatorname{det} R(\xi))^{1 / n}$ with respect to $\xi$. This can be done, e.g. numerically, using the LevenbergMarquardt algorithm with bounds for the components of $\xi$.

For numerical minimisation one needs the first and the second order derivatives of $h(\xi)$ with respect to $t, p$ (components of vector $\xi$ ), which can be found using expressions (18):

$$
\begin{aligned}
& \frac{\partial \widehat{\sigma}^{2}}{\partial t}=-\frac{1}{n} V_{s}^{T} \frac{\partial R}{\partial t} V_{s} \\
& \begin{aligned}
\frac{\partial^{2} \widehat{\sigma}^{2}}{\partial t \partial p}=\frac{2}{n} V_{s}^{T} \frac{\partial R}{\partial t} U \frac{\partial R}{\partial p} V_{s}-\frac{1}{n} V_{s}^{T} \frac{\partial^{2} R}{\partial t \partial p} V_{s} \\
\frac{\partial}{\partial t}(\operatorname{det} R)^{1 / n}=\frac{1}{n}(\operatorname{det} R)^{1 / n} \operatorname{tr}\left(R_{t}\right) \\
\frac{\partial^{2}}{\partial t \partial p}(\operatorname{det} R)^{1 / n}=\frac{1}{n}(\operatorname{det} R)^{1 / n} \\
\cdot\left\{\frac{1}{n} \operatorname{tr}\left(R_{t}\right) \operatorname{tr}\left(R_{p}\right)-\operatorname{tr}\left(R_{t} R_{p}\right)+\operatorname{tr}\left(R_{t p}\right)\right\}
\end{aligned}
\end{aligned}
$$

where $\quad U=R^{-1}-R^{-1} F\left(F^{T} R^{-1} F\right)^{-1} F^{T} R^{-1}, \quad V_{s}=U Y_{s}$, $R_{t}=R^{-1} \frac{\partial R}{\partial t}, R_{p}=R^{-1} \frac{\partial R}{\partial p}, R_{t p}=R^{-1} \frac{\partial^{2} R}{\partial t \partial p}$. Also, in this notation, it is convenient to write $\widehat{\sigma}^{2}=Y_{s}^{T} V_{s} / n$. Derivatives of $R$ with respect to $t, p$ essentially involve the derivatives of the $1 \mathrm{D}$ covariance $c(\cdot)$, and are easy to find.

If one uses LAPACK library for matrix calculations, a simple way of computing $R^{-1}$ is via Cholesky decomposition of the matrix. However, this method may occasionally fail if $R$ is badly conditioned. A more numerically stable approach found was to use DSYTRF procedure from LAPACK for factorization of symmetric indefinite matrices.

\section{Training the kriging proxy based on the gradients values}

Kriging proxy model constructed from a Gaussian process can be trained not only based on the values of the function $g(\cdot)$ at the design points, but also based on the derivatives, e.g. see $[11,24,36]$. In our work, we only consider the first derivatives for kriging proxy training, outlining below the main differences with the standard kriging scheme discussed so far. The approach of training on the derivative values relies on the property that the derivatives $\frac{\partial}{\partial x^{k}} \boldsymbol{Y}(x)$ of the Gaussian process $\boldsymbol{Y}(x)$ are also Gaussian processes. Denoting as before $x_{1}, \ldots$, $x_{n}$ the design points, $x$ the new point where the proxy should be calculated, and keeping other previous notation, introduce the following extended matrices and vectors: 


$$
\begin{gathered}
\bar{Y}_{(n+d n) \times 1}=\left(\begin{array}{c}
Y \\
\nabla \boldsymbol{Y}\left(x_{1}\right) \\
\vdots \\
\nabla \boldsymbol{Y}\left(x_{n}\right)
\end{array}\right), \bar{F}_{(n+d n) \times T}=\left(\begin{array}{c}
F \\
\nabla f^{T}\left(x_{1}\right) \\
\vdots \\
\nabla f^{T}\left(x_{n}\right)
\end{array}\right), \\
\bar{R}_{(n+d n) \times(n+d n)}=\left(\begin{array}{cccc}
R & \cdots & \bar{R}_{0, j} & \cdots \\
\vdots & \ddots & & \\
\bar{R}_{i, 0} & & \bar{R}_{i, j} & \\
\vdots & & & \ddots
\end{array}\right)
\end{gathered}
$$

where $\bar{Y}$ - random vector of values of the Gaussian process and its gradients at the design points, $\bar{F}$ - values and gradients of the trend functions at the design points, $R$ - correlation matrix for $\bar{Y}$. The submatrix blocks of $\bar{R}$ have obvious meaning which follows from the structure of $\bar{Y}$ : block $\bar{R}_{0, j}=\frac{1}{\sigma^{2}} \operatorname{Cov}\left(Y, \nabla \boldsymbol{Y}\left(x_{j}\right)\right)$, block $\bar{R}_{i, 0}=\frac{1}{\sigma^{2}} \operatorname{Cov}\left(\nabla \boldsymbol{Y}\left(x_{i}\right), Y\right), \operatorname{block} \bar{R}_{i, j}=\frac{1}{\sigma^{2}} \operatorname{Cov}\left(\nabla \boldsymbol{Y}\left(x_{i}\right), \nabla \boldsymbol{Y}\left(x_{j}\right)\right)$, where $i, j=1, \ldots, n$. Also, introduce $\bar{r}(x)_{(n+d n) \times 1}=\frac{1}{\sigma^{2}} \operatorname{Cov}$ $(y(x), \bar{Y})^{T}$ vector of correlations between the GP at $x$ and $\bar{Y}$. Quantities $y(x), f(x), \beta$ remain as before, and do not have the barred counterparts.

The differential operator involved in $\bar{Y}$ is linear and exchangeable with expectation and covariance. So, given the notation, the mean of the random vector $\bar{Y}$ is $\bar{F} \beta$. The correlation matrix $\bar{R}$ is calculated using the following two relationships:

$$
\begin{aligned}
& \operatorname{Cov}\left(\frac{\partial}{\partial x_{i}^{k}} \boldsymbol{Y}\left(x_{i}\right), \boldsymbol{Y}\left(x_{j}\right)\right) \\
& =\frac{\partial}{\partial x_{i}^{k}} \operatorname{Cov}\left(\boldsymbol{Y}\left(x_{i}\right), \boldsymbol{Y}\left(x_{j}\right)\right)=\frac{\partial}{\partial x_{i}^{k}} \sigma^{2} R_{i, j} \\
& \operatorname{Cov}\left(\frac{\partial}{\partial x_{i}^{k}} \boldsymbol{Y}\left(x_{i}\right), \frac{\partial}{\partial x_{j}^{l}} \boldsymbol{Y}\left(x_{j}\right)\right) \\
& =\frac{\partial^{2}}{\partial x_{i}^{k} \partial x_{j}^{l}} \operatorname{Cov}\left(\boldsymbol{Y}\left(x_{i}\right), \boldsymbol{Y}\left(x_{j}\right)\right)=\frac{\partial^{2}}{\partial x_{i}^{k} \partial x_{j}^{l}} \sigma^{2} R_{i, j}
\end{aligned}
$$

where $x_{i}^{k}$ denotes $k$-th component of $i$-th design point, and $R_{i, j}$ are elements of the correlation matrix $R$, as before. Applying (20), we can find that submatrix $R_{i, 0}$ has $j$-th column equal to $c^{\prime}\left(r_{i j} / r\right) /\left(r r_{i j}\right) \cdot\left\{x_{i}-x_{j}\right\}, i, j=1, \ldots n$, where $r_{i j}=$ $\left\|x_{i}-x_{j}\right\|$. Submatrices $\bar{R}_{0, j}$ are defined symmetrically. Applying (21), submatrices $\bar{R}_{i, j}$ are given by

$$
\begin{aligned}
\bar{R}_{i, j}=- & \frac{1}{r_{i j}^{2}}\left[\frac{c^{\prime \prime}\left(r_{i j} / r\right)}{r^{2}}-\frac{c^{\prime}\left(r_{i j} / r\right)}{r r_{i j}}\right] . \\
& \cdot\left(x_{i}-x_{j}\right)\left(x_{i}-x_{j}\right)^{T}-\frac{c^{\prime}\left(r_{i j} / r\right)}{r r_{i j}} \cdot I_{d \times d}
\end{aligned}
$$

Finally, the first subvector of $\bar{r}(x)$ is just the $r(x)_{n \times 1}$ defined previously within the gradient-free approach. The other subvectors of $\bar{r}(x)$ corresponding to $\operatorname{Cov}\left(y(x), \nabla \boldsymbol{Y}\left(x_{i}\right)\right)^{T}$ are found, using $(20)$, as $c^{\prime}\left(r_{x i} / r\right) /\left(r r_{x i}\right) \cdot\left\{x_{i}-x\right\}, i=1, \ldots n$, where $r_{x i}=\left\|x-x_{i}\right\|$.

With all the quantities above describing the extended vector $\bar{Y}$, we can build the kriging estimate in the way it was done in (12), (13), (14), replacing $Y, F, R, r(x)$ by $\bar{Y}, \bar{F}, \bar{R}, \bar{r}(x)$ respectively. The value of the kriging proxy is provided by the mean of the conditional distribution of $y(x)$ given $\bar{Y}=\bar{Y}_{s}$ :

$$
\begin{aligned}
G(x) \equiv \mathbb{E}\left(y(x) \mid \bar{Y}=\bar{Y}_{s}\right)= \\
\\
\quad f^{T}(x) \widehat{\bar{\beta}}+\bar{r}^{T}(x) \bar{R}^{-1}\left(\bar{Y}_{s}-\bar{F} \widehat{\bar{\beta}}\right),
\end{aligned}
$$

where $\bar{Y}_{s}=\left(Y_{s}^{T}, \nabla g\left(x_{1}\right)^{T}, \ldots, \nabla g\left(x_{n}\right)^{T}\right)^{T}$, a n d $\widehat{\bar{\beta}}=$ $\left(\bar{F}^{T} \bar{R}^{-1} \bar{F}\right)^{-1} \bar{F}^{T} \bar{R}^{-1} \bar{Y}_{s}$.

Note that the approach described above easily allows the set of design points where the function values are given, and the set of design points where the gradients are given, to be different sets. Besides, it is simple to implement kriging proxy training based only on the subset of components of the gradient, rather than the full gradient vector.

We also note that the size of kriging matrix grows fast when information from the gradients is supplied to the kriging proxy, especially when the dimension of the parameters space $d$ is high. Too large a size of the kriging matrix (say, larger than $4000 \times 4000$ ) may slow down the proxy computations significantly, making it difficult to adopt the gradient information from the sufficient number of design points.

Finally, in the situation where the kriging proxy is built for a simulator of some physical system, e.g. a reservoir simulator, the gradients may be available with reasonable efficiency only for the whole objective function. The calculation of the gradients of different modelled data (the sensitivity matrix) may have notably higher computational cost. Having only the objective function gradients, one may train the simple proxy, but not the data proxy (which is generally more accurate), as the latter would require the full sensitivity matrix for all the simulator outputs involved in the objective function.

\section{Data proxy}

Data proxy $G(x)$ involves building sub-proxies for the different simulator outputs, and then combining them into a single objective function (misfit, or log likelihood):

$$
G(x)=\left(\tilde{d}(x)-d_{o}\right)^{T} C^{-1}\left(\tilde{d}(x)-d_{o}\right),
$$


where for each component $d_{i}(x)$ of the simulator output vector $d(x)$, a usual kriging proxy $\widetilde{d}_{i}(x)$ is constructed, forming then the vector $\tilde{d}(x)$. The gradient of the data proxy $G(x)$ can be found as:

$\nabla G(x)=2 \cdot D^{T}(x) C^{-1}\left(\tilde{d}(x)-d_{o}\right)$

where matrix $D^{T}(x)$ has $i$-th column equal to $\nabla \widetilde{d}_{i}(x)$. The Fisher Information matrix for the likelihood defined as $\exp \left(-\frac{1}{2} G(x)\right)$ can be found by

$\mathrm{FI}(x)=D^{T}(x) C^{-1} D(x)$.

As can be seen, individual sub-proxies can be built and used independently, allowing parallel calculations for the data proxy. For example, when calculating (24) in parallel, the subproxies are distributed between the parallel processes, and each process calculates its own chunk of vector $\widetilde{d}(x)$. When vector $\tilde{d}(x)$ has been found, the results are gathered to find the final value $G(x)$. The same principles are employed when calculating in parallel the gradients and other quantities.

\section{Proxy training scheme}

The choice of the design points where the kriging proxy should be trained is a subject of design of computer experiments. In terms of implementation, the simplest designs are the space filling designs, e.g. uniform design and Latin hypercube design. In our work, we use the uniform Sobol sequence design to perform the initial training of the proxy model. The initial ensemble cannot be too big because the simulator should be run for all the points in it.

The space filling designs may generate points in the unnecessary regions of the parameter space, leading to the wasted computations. In this situation, adaptive designs are more appropriate. The adaptive designs can be subdivided into singlestage designs (optimal selection of multiple points at a time), and sequential designs (usually taking one point at a time) which are not optimal, but efficient. Some examples of sequential designs are: Active Learning MacKay (ALM), Active Learning Cohn (ALC), and Mutual Information for Computer Experiments (MICE), the latter proposed by Beck and Guillas [3]. A more comprehensive overview of the sequential design literature can be found in the short article [14].

In our work, we use a simpler approach. In addition to the initial Sobol sequence, a heuristic method selects a subset from the points visited by the MCMC sampler during the burn-in stage (at these points the simulator data are readily available). The need to select a subset of the visited points arises from the limitation on the total number of design points a kriging proxy can handle. Essentially, a feasible kriging proxy is limited to a maximum of a few thousand design points (1000-5000) - this number comes from the dimension of the dense linear system (15) which can be solved in a reasonable time.

The points visited by the sampler during the burn-in (both accepted and rejected ones) are collected into the candidate set $K$. At every $n_{K}$-th iteration of the sampler, the proxy update event takes place: firstly, a subset is selected from $K$ and added to the main design points set $C$. Next, the proxy model is retrained based on $C$.

The heuristic sequential approach used for selecting the subset from $K$ works as follows. Given the current set of design points $C=\left\{x_{1}, \ldots, x_{c}\right\}$, we find the candidate point $x^{*} \in K$ which minimises the sum $1 /\left\|x_{1}-x^{*}\right\|^{3}+\ldots+1 /\left\|x_{c}-x^{*}\right\|^{3}$, and add this point to $C$. Then, the procedure is repeated until the required number of points have been added to $C$. The motivation for this algorithm is to get the points which have reasonably high separation from each other.

Furthermore, we consider two variants of this procedure, referred to as update strategy 0 and update strategy 1 . For both of them, the burn-in stage of the MCMC is split into $n_{I}$ epochs, each consisting of $n_{K}$ iterations forming the set $K_{i}$ of size $n_{K}$, $i=1, \ldots, n_{I}$; so, the whole set of burn-in samples can be written as $K_{1} \cup \ldots \cup K_{n_{I}}$. For strategy 0 , the $i$-th proxy update event happens in the end of epoch-i, working as follows:

- $\quad$ Take the candidate set $K=K_{i}$ (it has been visited by the sampler in the current epoch),

- Iteratively select $n_{C}$ points from $K$, adding them to the main points set $C$, which progressively grows,

- $\quad$ Re-train the proxy based on $C$.

Thus, after all $n_{I}$ epochs, $n_{I} \cdot n_{C}$ points will have been added to $C$ in total. The typical examples of the constants used in our work are $n_{I}=100, n_{C}=15, n_{K}=50$.

Update strategy 1 does not gradually accumulate the design points set $C$, but instead only keeps the initial (Sobol) design points $C_{0}$. At the $i$-th proxy update event, which is again in the end of epoch- $i$, do:

- $\quad$ Set $C=C_{0}$,

- $\quad$ Take the accumulated candidate set $K=K_{1} \cup \ldots \cup K_{i}$ (these points have been visited by the sampler in all epochs, including the current one),

- $\quad$ Iteratively select $i \cdot n_{C}$ points from $K$, adding them to the main points set $C$,

- Re-train the proxy based on $C$.

As in the update strategy 0 , after all $n_{I}$ proxy updates, $n_{I} \cdot n_{C}$ points will have been added to $C$ in total. Update strategy 1 may work notably slower than strategy 0 because of performing point selections from the growing candidate set.

The motivation for adopting the heuristic strategy 1 is in improving the design efficiency for the cases where the 
Markov chain starts from a very slow exploration of the parameter space, gradually accelerating. The initial slow part of the chain will produce the closely spaced design points, which may be excessive and unnecessary for the proxy model. Update strategy 0 has to take a certain number of points in each epoch of the burn-in stage, including the initial slow segment. However, strategy 1 , once it has passed the slow segment, can select points from the accumulated candidate set, and has freedom to avoid the closely spaced initial points.

\section{Appendix B. Domain boundaries in HMC}

In all the HMC sampling problems we consider in our work, the parameter space is bounded to a rectangular region defined by $l_{i} \leq$ $x_{i} \leq u_{i}$. When the point trajectory crosses the boundary during the Hamiltonian dynamics move, some action should be taken in order for the point to remain in the feasible region. A natural choice is performing some sort of reflection every time a leapfrog coordinate update $x(t) \rightarrow x(t+\varepsilon)$ crosses the boundary at some point $x^{\prime}$. To do a reflection, first re-write the second equation from (5) using velocity $v=M^{-1} p$ :

$x(t+\varepsilon)=x(t)+\varepsilon v(t+\varepsilon / 2)$

At the boundary intersection point $x^{\prime}$ corresponding to the partial time $\operatorname{step} \varepsilon^{\prime}$, we perform velocity reflection: $v \rightarrow v^{\prime}$, and then coordinate update step (27) is resumed for the remaining time step: $x(t+\varepsilon)=x^{\prime}+\left(\varepsilon-\varepsilon^{\prime}\right) v^{\prime}$. Velocity reflection in our work is done by applying a reflection matrix: $v^{\prime}=R_{i} \cdot v$, where

$R_{i}=I-2 \frac{M^{-1} e_{i} e_{i}^{T}}{e_{i}^{T} M^{-1} e_{i}}$

$i$ is the index of the coordinate for which the boundary crossing took place, i.e. the index for which $x_{i}^{\prime}=l_{i}$ or $x_{i}^{\prime}=u_{i}$, and $e_{i}$ is the $i$-th cartesian coordinate basis vector. Velocity reflection does not change the potential energy by definition. Kinetic energy $\frac{1}{2} p^{T} M^{-1} p=\frac{1}{2} v^{T} M v$ also does not change if $v$ is replaced by $R_{i} v$ since one can easily establish that $R_{i}^{T} M R_{i}=M$. Next, for the reflection matrix it holds that $R_{i}^{2}=I$, which means that $\operatorname{det} R_{i}= \pm 1$, and the mapping $v \rightarrow v^{\prime}$ is volume-preserving. The velocity reflection step also preserves the reversibility of the Hamiltonian dynamics move: consider reflection $v^{\prime}=R_{i} v$, and start moving in the opposite direction $-v^{\prime}$ performing one more reflection $v^{\prime \prime}=R_{i}\left(-v^{\prime}\right)=-R_{i}^{2} v=-v$, which is exactly the reversed original direction. Furthermore, examine how reflection matrix $R_{i}$ affects $i$-th component of the velocity vector, by considering the value of $e_{i}^{T} v$ before and after reflection. It can be easily found that after reflection $e_{i}^{T}\left(R_{i} v\right)=-e_{i}^{T} v$, so the $i$-th velocity component simply changes sign. This also means that after applying the reflection, the velocity vector will point back to the target rectangular region, and the point will not escape from that region. Finally, we note that for diagonal mass matrices $M$, the reflection matrix $R_{i}$ becomes equal to $W_{i} \equiv I-2 e_{i} e_{i}^{T}$, a matrix that changes sign of component $i$ of a vector, and does not change the other components. $W_{i}$ corresponds to the ordinary bouncing of a particle from a wall. However, if the mass matrix is not diagonal, the simple bouncing will not preserve the kinetic energy, since $W_{i}^{T} M W_{i} \neq M$, and employing $W_{i}$ for reflections in HMC would result in rather high changes in the total energy after leap frog integration, making the sampler inefficient.

The Hamiltonian dynamics move with reflections defined via matrix $R_{i}$ will remain time-reversible, volume-preserving, and will preserve energy with order $O\left(\varepsilon^{2}\right)$, so the HMC sampler remains well defined.

\section{Appendix C. Proxy settings for different examples}

Rosenbrock 5D This problem is simple, and the proxy model becomes accurate quite easily, so a small number of design points turned out to suffice. Initial design points: 5 points from Sobol sequence +1 starting point. Additional design points taken during burn-in: 1 point for every 10 iterations. Onedimensional covariance: Matérn with $\nu=5$, correlation radius $r=8$, linear trend.

Punq-S3 This problem is quite hard, and the proxy model does not become sufficiently accurate at all. So, we tried to take as many design points as kriging can handle in reasonable time. Initial design points: 100 points from Sobol sequence +1 starting point. Additional design points taken during burn-in: 15 points for every 50 iterations, resulting in 1601 design points in the end of burn-in. Update strategy 1 was used. One-dimensional covariance: Matérn with $\nu=3.5$, correlation radius from $r=2$ (6-dimensional case) to $r=4$ (31-dimensional case), constant trend.

Synthetic single-phase reservoir with $\mathbf{4 0}$ wells Like Punq-S3 simulation, this challenging problem required as many design points as possible. Initial design points: 1500 points from Sobol sequence +1 starting point. Additional design points taken during burn-in: 5 points for every 10 iterations, resulting in 3001 design points in the end of burn-in. (For the simple proxy trained with gradients: 75 initial Sobol points +1 starting point, followed by 1 point every 40 iterations, resulting in 151 design points after burn-in).

Update strategy 1 was used (except one case with data proxy, where strategy 0 was used). One-dimensional 
covariance: Matérn with $\nu=5$, correlation radius $r=4$, constant trend.

Open Access This article is licensed under a Creative Commons Attribution 4.0 International License, which permits use, sharing, adaptation, distribution and reproduction in any medium or format, as long as you give appropriate credit to the original author(s) and the source, provide a link to the Creative Commons licence, and indicate if changes were made. The images or other third party material in this article are included in the article's Creative Commons licence, unless indicated otherwise in a credit line to the material. If material is not included in the article's Creative Commons licence and your intended use is not permitted by statutory regulation or exceeds the permitted use, you will need to obtain permission directly from the copyright holder. To view a copy of this licence, visit http://creativecommons.org/licenses/by/4.0/.

\section{References}

1. Punq-S3 model. Available from: https://www.imperial.ac.uk/earthscience/research/research-groups/perm/standard-models/

2. Abdollahzadeh A, Reynolds A, Christie M, Corne D, Davies B, and Williams G, (2011) Bayesian optimization algorithm applied to uncertainty quantification, in SPE-143290-MS

3. Beck, J., Guillas, S.: Sequential design with mutual information for computer experiments (MICE): emulation of a tsunami model. SIAM/ASA J Uncertain Quantif. 4(1), 739-766 (2016)

4. Betancourt M, Byrne S, and M. Girolami, (2015) Optimizing the integrator step size for Hamiltonian Monte Carlo. arXiv

5. Bilionis, I., Zabaras, N.: Multi-output local Gaussian process regression: applications to uncertainty quantification. J Comput Phys. 231, 5718-5746 (2012)

6. Bou-Rabee N and Sanz-Serna JM, Geometric integrators and the Hamiltonian Monte Carlo method. Acta Numerica, 2018: p. 1-92

7. Bui-Thanh, T., Ghattas, O., Higdon, D.: Adaptive Hessian-based nonstationary Gaussian process response surface method for probability density approximation with application to Bayesian solution of largescale inverse problems. SIAM J Sci Comput. 34, A2837-AA287 (2011)

8. Busby, D., Da Veiga, S., Touzani, S.: A workflow for decision making under uncertainty. Comput Geosci. 18, 519-533 (2014)

9. Christen, J.A., Sanso, B.: Advances in the sequential design of computer experiments based on active learning. Commun Statistics - Theory Methods. 40, 4467-4483 (2011)

10. Conrad, P., Marzouk, Y., Pillai, N., Smith, A.: Accelerating asymptotically exact MCMC for computationally intensive models via local approximations. J Am Stat Assoc. 111, 1591-1607 (2015)

11. Eriksson, D., K. Dong, E.H. Lee, D. Bindel, and A.G. Wilson. Scaling Gaussian process regression with derivatives. in Neural information processing systems 2018

12. Floris, F.J.T., Bush, M.D., Cuypers, M., Roggero, F., Syversveen, A.-R.: Methods for quantifying the uncertainty of production forecasts: a comparative study. Pet Geosci. 7, S87-S96 (2001)

13. Geyer, C.J.: Practical Markov chain Monte Carlo. Stat Sci. 7(4), 473-483 (1992)

14. Ginsbourger, D., Sequential design of computer experiments. Wiley StatsRef, 2017

15. Girolami, M., Calderhead, B.: Riemann manifold Langevin and Hamiltonian Monte Carlo methods. J R Stat Soc. 73, 123-159 (2011)

16. Gramacy, R.B., Lee, H.K.H.: Bayesian treed Gaussian process models with an application to computer modeling. J Am Stat Assoc. 103, 1119-1130 (2008)
17. Gramacy, R.B. and Lee, H.K.H, Cases for the nugget in modeling computer experiments. arXiv paper, 2010

18. Hajizadeh, Y., Ants can do history matching, in SPE-141137-STU. 2010

19. He, J., J. Xie, X.-H. Wen, and W. Chen, Improved proxy for history matching using proxy-for-data approach and reduced order modeling, in SPE-174055-MS. 2015

20. Hoffman, M.D., Gelman, A.: The no-U-turn sampler: adaptively setting path lengths in Hamiltonian Monte Carlo. J Mach Learn Res. 15, 1351-1381 (2014)

21. Jones, D.R.: A taxonomy of global optimization methods based on response surfaces. J Glob Optim. 21, 345-383 (2001)

22. Kaipio, J. and E. Somersalo, Statistical and computational inverse problems. 2005: Springer

23. Korattikara, A., Y. Chen, and M. Welling. Austerity in MCMC land: cutting the Metropolis-Hastings budget. in 31st International Conference on Machine Learning. 2014

24. Lan, S., Bui-Thanh, T., Christie, M., Girolami, M.: Emulation of higher-order tensors in manifold Monte Carlo methods for Bayesian inverse problems. J Comput Phys. 308, 81-101 (2016)

25. Lan, S., Stathopoulos, V., Shahbaba, B., Girolami, M.: Markov chain Monte Carlo from Lagrangian dynamics. J Comput Graph Stat. 24(2), 357-378 (2015)

26. Landa, J. and G. Baris, A methodology for history matching and the assessment of uncertainties associated with flow prediction, in SPE ATCE. 2003

27. Liu, J.S., Monte-Carlo strategies in scientific computing. Springer Series in Statistics. 2004: Springer; 1st edition

28. Ma, Y., E.B. Fox, T. Chen, and L. Wu, Irreversible samplers from jump and continuous Markov processes. arXiv, 2018

29. Neal, R.M., MCMC using Hamiltonian dynamics, in Handbook of Markov Chain Monte Carlo, S. Brooks, et al., Editors. 2011, Chapman \& Hall / CRC Press

30. Oakley, J., Bayesian uncertainty analysis for complex computer codes. 1999, University of Sheffield

31. Oliver, D.S., A.C. Reynolds, and N. Liu, Inverse theory for petroleum reservoir characterization and history matching. 2008: Cambridge University Press

32. Osborne, M.A., R. Garnett, and S.J. Roberts. Gaussian processes for global optimization. in 3rd International conference on learning and intelligent optimization. 2009

33. Rasmussen, C.: Gaussian processes to speed up hybrid Monte Carlo for expensive Bayesian integrals. Bayesian Statistics. 7, 651-659 (2003)

34. Robert, C. and G. Casella, Monte Carlo Statistical Methods. 2 ed. 2004: Springer Science \& Business Media

35. Sacks, J., Welch, W.J., Mitchell, T.J., Wynn, H.P.: Design and analysis of computer experiments. Stat Sci. 4(4), 409-423 (1989)

36. Solak, E., R. Murray-Smith, W.E. Leithead, D.J. Leith, and C.E. Rasmussen. Derivative observations in Gaussian process models of dynamic systems. in Neural Information Processing Systems. 2002

37. Stein, M.L.: Interpolation of spatial data: some theory for kriging. Springer, New York (1999)

38. Tarantola, A., Inverse problem theory and methods for model parameter estimation. 2005

39. Welling, M. and Y. Teh. Bayesian learning via stochastic gradient Langevin dynamics. in 28th International Conference on Machine Learning. 2011

40. Zubarev, D.I., Pros and cons of applying proxy-models as a substitute for full reservoir simulations, in SPE-124815-MS. 2009

Publisher's note Springer Nature remains neutral with regard to jurisdictional claims in published maps and institutional affiliations. 